\title{
Analysis of a vaccine model with cross-immunity: when can two competing infectious strains coexist?
}

\author{
I. Dorigatti*a , A. Pugliese ${ }^{a}$
}

May 18, 2011

a Department of Mathematics, University of Trento, via Sommarive 14, 38123 Povo

(Tn), Italy

* Corresponding Author. Email: dorigatti@science.unitn.it phone: +390461283983 fax: +390461281624

\begin{abstract}
We analyse here the Vaccine Model with Cross-Immunity proposed by Porco and Blower [1]. Porco and Blower[1] show that vaccination can shift the competitive balance in favour of a strain that, without vaccination, would be out-competed and that vaccination can also promote coexistence of different strains, something that normally is not expected [2]. Their results have been mainly obtained through numerical simulations, so that the conditions under which a shift in competitive balance or coexistence occurs have not been fully established. We give a rather complete description of its behavior, at least in terms of equilibria. We find the exact conditions under which vaccination may lead to a shift in competitive balance and show that, under these conditions, there always exist a range of vaccination rates under which a coexistence equilibrium exists. We also find that a coexistence equilibrium exists (and is unstable) in a 'bi-stability' region, where both
\end{abstract}


monomorphic equilibria are stable. This fact has been rarely observed in models of competition between pathogen strains.

Keywords: Vaccine Model, HIV, Cross-Immunity, Vaccination Rate, Coexistence Equilibrium, Forward/Backward Bifurcation

\section{Introduction}

Control policies of infectious diseases can lead to unexpected outcomes when the infectious agents consist of a variety of different strains. In fact, it has often be argued that more pathogenic strains are in competition with less pathogenic ones [2], so that the application of control policies may shift the competitive balance in favour of the less fit strains $[3,4]$ that might however be more virulent.

It has been observed in previous studies and in practice that vaccination, one of the most powerful control policies, can have very dramatic effect on the outcome of the competition between more pathogens. This topic has been examined with the use of mathematical models in several papers $[1,5,6,7]$. Vaccination can destabilize the existing host-pathogen evolutionary equilibria, accelerate pathogen evolution and also lead to the emergence or dominance of a once-rare pathogen, a mechanism also known as strain replacement $[8,6,7]$. Vaccines differ for their mode of action; vaccines with differential effectiveness provide different degrees of protection against infection by the different strains of the pathogen and their efficacy has been extensively discussed in the literature $[9,10,11,7]$.

Porco and Blower [1] showed that vaccination can indeed shift the competitive balance in favour of a strain that, without vaccination, would be out-competed and that vaccination can also promote coexistence of different strains, something that normally is not expected [2]. The results by Porco 
and Blower have been mainly obtained through numerical simulations and only few analytical results have been obtained: these are explicitly recalled at the beginning of Section 3 of this manuscript. In the original paper [1] the complete conditions under which a shift in competitive balance or coexistence occurs had not been fully established and here we aim to fill this gap. With this work we investigate and get a better understanding of the potential consequences of introducing a vaccine with differential effectiveness against an infectious agent circulating in multiple strains within a population, something that could be well suited to many sexually transmitted infections like HIV, HPV (Human Papilloma Virus) and HSV (Herpes Simplex Virus), although the model examined has been specifically designed for HIV.

Here we examine in detail the "Vaccine Model with Cross-Immunity" or "Differential Degree Model" proposed in Porco and Blower [1] to describe the spread of 2 HIV strains and the subsequent progression into AIDS in a population of potential sex partners. More in general, the model can be thought as describing the spread of two competing pathogens within a population in presence of vaccination and cross-immunity. We analyse the impact of vaccination at the community level and give a rather complete description of the model behavior, at least in terms of equilibria. We find the exact conditions under which vaccination may lead to a shift in competitive balance and also show that, under these conditions, there always exist a range of vaccination rates under which a coexistence equilibrium exists. We find that the Coexistence Equilibrium may be stable or unstable, depending on another condition. The former case corresponds to what had already been observed numerically. In the latter case, the parameter region in which a coexistence equilibrium exists is actually a 'bi-stability' region in 
which both monomorphic equilibria are stable, so that asymptotic behavior depends on initial conditions. This fact, that would lead to a sort of hysteresis cycle if vaccination rates were increased then decreased, has rarely been demonstrated in models of competition between pathogen strains.

\section{Model Formulation}

The "Vaccine Model with Cross-Immunity" proposed by Porco and Blower [1] is a particular transmission dynamics model of HIV in presence of two subtypes and a vaccine that provides a degree of protection against infection by both subtypes.

The state variables are $X$ (the number of susceptible individuals), $V$ (the number of effectively vaccinated individuals), $Y_{1}$ and $Y_{2}$ (the number of individuals infected with subtype 1 and subtype 2 respectively and have not developed AIDS), $A_{1}$ and $A_{2}$ (the number of individuals who have been infected with subtype 1 and subtype 2 respectively and have developed AIDS). The state variables are supposed to be $C^{1}$ functions of the time variable $t$. Individuals are part of a community of potential sex partners and we assume that individuals with AIDS do not acquire new sex partners. This means that the sexually active community $N$ is given by $N=X+V+Y_{1}+Y_{2}$. We assume that individuals enter the community at a constant rate $\pi$ and a fraction $p$ of these are vaccinated. The vaccine induces a protective immune response in a fraction $e$ of the vaccinated individuals, that is the vaccine takes only in a fraction pe of the new entries. Uninfected individuals either not vaccinated or who were vaccinated but in whom the vaccine did not take, are referred as being completely susceptible. The degree of protection

conferred by the vaccine against subtype $\mathrm{i}$ is indicated with $\xi_{i}\left(0 \leqslant \xi_{i} \leqslant 1\right)$; $\xi_{i}=0$ corresponds to no protection and $\xi_{i}=1$ corresponds to complete 
protection against infection. Individuals leave each class at a constant per capita rate $\mu$ when they cease acquiring new sex partners. The transmission probability of subtype i per partnership is indicated with $\beta_{i}$, the number of new sex partners per unit time is indicated by $c, \gamma_{i}$ is for the rate of progression to AIDS and $\alpha$ indicates the death rate due to AIDS.

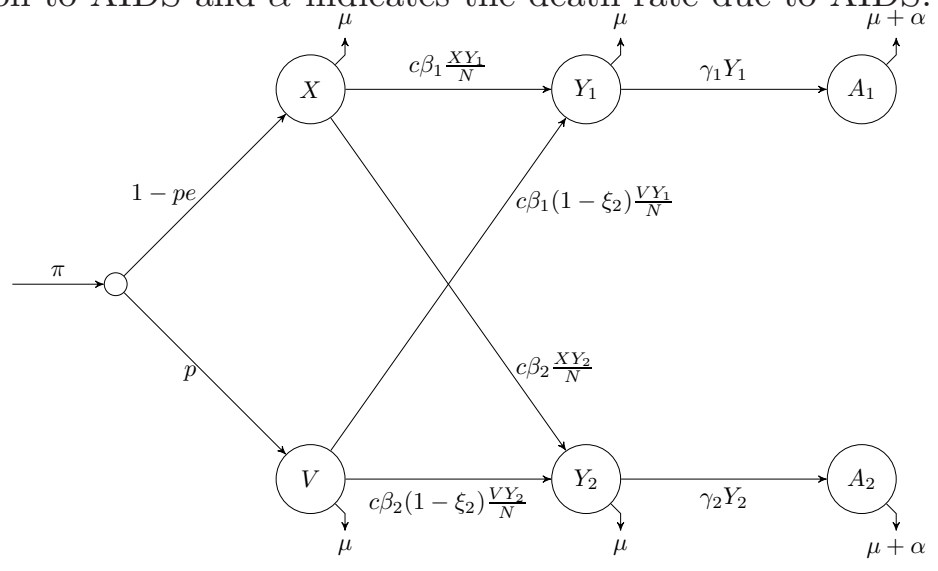

Figure 1: The flow chart of the model

The flow diagram in Figure 1 describes the dynamics of the "Vaccine Model with Cross-Immunity". The differential equations describing the corresponding dynamics are:

$$
\begin{aligned}
\dot{X} & =\pi(1-p e)-\mu X-c \beta_{1} X \frac{Y_{1}}{N}-c \beta_{2} X \frac{Y_{2}}{N} \\
\dot{V} & =\pi p e-\mu V-\left(1-\xi_{1}\right) c \beta_{1} V \frac{Y_{1}}{N}-\left(1-\xi_{2}\right) c \beta_{2} V \frac{Y_{2}}{N} \\
\dot{Y}_{1} & =c \beta_{1} X \frac{Y_{1}}{N}+\left(1-\xi_{1}\right) c \beta_{1} V \frac{Y_{1}}{N}-\left(\mu+\gamma_{1}\right) Y_{1} \\
\dot{Y}_{2} & =c \beta_{2} X \frac{Y_{2}}{N}+\left(1-\xi_{2}\right) c \beta_{2} V \frac{Y_{2}}{N}-\left(\mu+\gamma_{2}\right) Y_{2} \\
\dot{A}_{1} & =\gamma_{1} Y_{1}-(\mu+\alpha) A_{1} \\
\dot{A}_{2} & =\gamma_{2} Y_{2}-(\mu+\alpha) A_{2}
\end{aligned}
$$

where $N=X+V+Y_{1}+Y_{2}$.

We observe that equations (1)-(4) are sufficient to describe the behavior 
of the system. Furthermore, these equations can be suitable for any infection of $S I$ type, where $\gamma_{1}$ and $\gamma_{2}$ denote disease-induced mortality rates, and vaccination occurs at birth. The assumption of a constant (independent of population size) input rate $\pi$ in the population may then need to be amended. We introduce the reproduction numbers [12]

$$
R_{0}^{1}=\frac{c \beta_{1}}{\mu+\gamma_{1}} \quad R_{0}^{2}=\frac{c \beta_{2}}{\mu+\gamma_{2}}
$$

Briefly, the reproduction number $R_{0}$ for a deterministic SIR-type model is a threshold condition that determines the emergence (if $R_{0}>1$ ) of an epidemic and represents the average number of cases generated by an infectious individual at the beginning of an epidemic (i.e. when the whole population is susceptible). In this work we are going to define different reproduction numbers, which for clarity have been also summarized on Table 1. Then, performing the change of variables

$$
x=\frac{X}{N} \quad v=\frac{V}{N} \quad y_{1}=\frac{Y_{1}}{N} \quad y_{2}=\frac{Y_{2}}{N}
$$

and using $R_{0}^{1}$ and $R_{0}^{2}$ as parameters, system (1)-(4) can be equivalently written as

$$
\begin{aligned}
\dot{x} & =\frac{\pi}{N}(1-x-p e)-x\left[\left(R_{0}^{1}\left(\mu+\gamma_{1}\right)-\gamma_{1}\right) y_{1}+\left(R_{0}^{2}\left(\mu+\gamma_{2}\right)-\gamma_{2}\right) y_{2}\right] \\
\dot{v} & =\frac{\pi}{N}(p e-v)-v\left[\left(R_{0}^{1}\left(\mu+\gamma_{1}\right)\left(1-\xi_{1}\right)-\gamma_{1}\right) y_{1}+\left(R_{0}^{2}\left(\mu+\gamma_{2}\right)\left(1-\xi_{2}\right)-\gamma_{2}\right) y_{2}\right](9) \\
\dot{y}_{1} & =y_{1}\left[R_{0}^{1}\left(\mu+\gamma_{1}\right)\left(x+\left(1-\xi_{1}\right) v\right)-\gamma_{1}\left(1-y_{1}\right)+\gamma_{2} y_{2}-\frac{\pi}{N}\right] \\
\dot{y}_{2} & =y_{2}\left[R_{0}^{2}\left(\mu+\gamma_{2}\right)\left(x+\left(1-\xi_{2}\right) v\right)-\gamma_{2}\left(1-y_{2}\right)+\gamma_{1} y_{1}-\frac{\pi}{N}\right] \\
\dot{N} & =\pi-N\left(\mu+\gamma_{1} y_{1}+\gamma_{2} y_{2}\right)
\end{aligned}
$$

In (8), we have dropped the dependency on $c, \beta_{1}$ and $\beta_{2}$ using instead the non-dimensional quantities $R_{0}^{1}$ and $R_{0}^{2}$ as parameters. It would be possible to 
reduce the parameters to a smaller number of non-dimensional quantities; we prefer to keep them all, while later showing that different behaviours depend on the ratios $\mu / \gamma_{i}$ and $\gamma_{1} / \gamma_{2}$.

By adding together (8)-(11) we get

$$
\dot{x}+\dot{v}+\dot{y}_{1}+\dot{y}_{2}=\left(\frac{\pi}{N}-\gamma_{1} y_{1}-\gamma_{2} y_{2}\right)\left[1-\left(x+v+y_{1}+y_{2}\right)\right]
$$

Since

$$
x+v+y_{1}+y_{2}=1
$$

is invariant for (8)-(11), as intuitively obvious, we can drop (for instance) the equation for $v$ and consider the system

$$
\left\{\begin{aligned}
\dot{N} & =\pi-N\left(\mu+\gamma_{1} y_{1}+\gamma_{2} y_{2}\right) \\
\dot{x} & =\frac{\pi}{N}(1-x-p e)-x\left[\left(R_{0}^{1}\left(\mu+\gamma_{1}\right)-\gamma_{1}\right) y_{1}+\left(R_{0}^{2}\left(\mu+\gamma_{2}\right)-\gamma_{2}\right) y_{2}\right] \\
\dot{y}_{1} & =y_{1}\left[R_{0}^{1}\left(\mu+\gamma_{1}\right)\left(x+\left(1-\xi_{1}\right)\left[1-\left(x+y_{1}+y_{2}\right)\right]\right)-\gamma_{1}\left(1-y_{1}\right)+\gamma_{2} y_{2}-\frac{\pi}{N}\right] \\
\dot{y}_{2} & =y_{2}\left[R_{0}^{2}\left(\mu+\gamma_{2}\right)\left(x+\left(1-\xi_{2}\right)\left[1-\left(x+y_{1}+y_{2}\right)\right]\right)-\gamma_{2}\left(1-y_{2}\right)+\gamma_{1} y_{1}-\frac{\pi}{N}\right] .
\end{aligned}\right.
$$

We can then obtain the fraction of vaccinated individuals by subtraction

$$
v=1-\left(x+y_{1}+y_{2}\right) .
$$

\section{$3 \quad$ Existence and Stability of Equilibria}

As mentioned above, Porco and Blower [1] present an extensive numerical analysis of the model, showing for instance the equilibrium coexistence of the strains for certain parameter values. From the analytical point of view they discuss the Disease Free Equilibrium and the Equilibrium with only one Subtype, thus finding

- the stability conditions of the Disease Free Equilibrium; 
- one necessary condition for the stability of The Subtype-1-Only Equilibrium (i.e. condition (24) in this manuscript);

- the fact that if $R_{0}^{1}>R_{0}^{2}>1$ and $\xi_{1} \leq \xi_{2}$, the Subtype-1-Only Equilibrium is asymptotically stable, whenever it exists.

In order to make our presentation smoother, we are going to present a complete analysis of the equilibria of the system, including the conditions already found by Porco and Blower [1].

We study here the equilibria of (14); when this makes the derivation shorter, we will consider also (8)-(11).

In the rest of the section we discuss in detail the conditions under which the equilibria exist and are stable. The reader can find a summary of the assumptions and the main results (in terms of model's behaviour) obtained in this section on Table 2.

Note first that, from (12), any steady state $\left(\tilde{x}, \tilde{v}, \tilde{y_{1}}, \tilde{y_{2}}, \tilde{N}\right)$ of (14) satisfies

$$
\frac{\pi}{\tilde{N}}=\mu+\gamma_{1} \tilde{y}_{1}+\gamma_{2} \tilde{y}_{2}
$$

The equilibria can be distinguished on the basis of whether $\tilde{y}_{1}$ and $\tilde{y}_{2}$ are zero or positive.

\subsection{Disease Free Equilibrium}

\subsubsection{Existence}

The Disease Free Equilibrium (DFE) occurs when the fraction of infected individuals is null $y_{1}^{\star}=y_{2}^{\star}=0$ and there are positive fractions of susceptible and vaccinated individuals $x^{\star} \neq 0, v^{\star} \neq 0$.

From (15), we obtain $N^{*}=\frac{\pi}{\mu}$. Setting the right-hand side of (8)-(9) 
equal to 0 with $y_{1}^{\star}=y_{2}^{\star}=0$, we immediately obtain for the DFE

$$
x^{\star}=1-p e \text { and } \quad v^{\star}=p e .
$$

This is always a feasible solution under the constraints $0 \leq p \leq 1,0 \leq e \leq 1$ arising from their definition. Otherwise said, the DFE always exists.

\subsubsection{Stability}

We study the local stability of the DFE through the Jacobian matrix of system (14) at the DFE $\left(x^{\star}, 0,0, N^{*}\right)=\left(1-p e, 0,0, \frac{\pi}{\mu}\right)$. The eigenvalues of the Jacobian at the DFE are

$$
\begin{gathered}
\lambda_{1}^{\star}=-\mu \\
\lambda_{2}^{\star}=-\mu \\
\lambda_{3}^{\star}=\left(\mu+\gamma_{1}\right)\left(R_{0}^{1}(1-p e)+\left(1-\xi_{1}\right) R_{0}^{1} p e-1\right) \\
\lambda_{4}^{\star}=\left(\mu+\gamma_{2}\right)\left(R_{0}^{2}(1-p e)+\left(1-\xi_{2}\right) R_{0}^{2} p e-1\right)
\end{gathered}
$$

Therefore, the DFE is stable if and only if $\lambda_{3}^{\star}<0$ and $\lambda_{4}^{\star}<0$.

Rearranging the terms, the necessary and sufficient conditions for the DFE to be stable can be written as

$$
\left\{\begin{array}{l}
R_{p}^{1}=R_{0}^{1}\left(x^{\star}+\left(1-\xi_{1}\right) v^{\star}\right)<1 \\
R_{p}^{2}=R_{0}^{2}\left(x^{\star}+\left(1-\xi_{2}\right) v^{\star}\right)<1 .
\end{array}\right.
$$

or

$$
\left\{\begin{array} { l } 
{ R _ { p } ^ { 1 } = R _ { 0 } ^ { 1 } ( 1 - \xi _ { 1 } p e ) < 1 } \\
{ R _ { p } ^ { 2 } = R _ { 0 } ^ { 2 } ( 1 - \xi _ { 2 } p e ) < 1 }
\end{array} \Longleftrightarrow \left\{\begin{array}{l}
p e>p e_{1}^{D F} \\
p e>p e_{2}^{D F}
\end{array}\right.\right.
$$

where

$$
\left\{\begin{array}{l}
p e_{1}^{D F}=\frac{R_{0}^{1}-1}{R_{0}^{1} \xi_{1}} \\
p e_{2}^{D F}=\frac{R_{0}^{2}-1}{R_{0}^{2} \xi_{2}} .
\end{array}\right.
$$


Observe that if $R_{0}^{1}\left(1-\xi_{1}\right)>1$ or $R_{0}^{2}\left(1-\xi_{2}\right)>1$, then the DFE is never stable.

Notice moreover that if

$$
\left\{\begin{array}{l}
R_{0}^{1}<1 \\
R_{0}^{2}<1
\end{array}\right.
$$

then the DFE is stable independently the choice of $p e, \xi_{1}$ and $\xi_{2}$.

For this reason we assume from now on that $R_{0}^{1}>1$ and $R_{0}^{2}>1$. Using these, in all the rest of the paper let us assume, without loss of generality, that

$$
R_{0}^{1}>R_{0}^{2}>1
$$

\subsection{Subtype-i-Only Equilibrium}

\subsubsection{Existence}

We analyse here the Subtype-1-Only Equilibrium.

By definition, at the Subtype-1-Only Equilibrium there are no individuals infected by subtype 2 (i.e. $\bar{y}_{2}=0$ ) and there are positive fractions of individuals infected by subtype $1\left(\bar{y}_{1}>0\right)$, susceptible $\left(\bar{x}_{1}>0\right)$ and vaccinated individuals $\left(\bar{v}_{1}>0\right)$.

Setting equal to 0 equation (10), together with (15) and $\bar{y}_{1}>0=\bar{y}_{2}$, one obtains

$$
R_{0}^{1}\left(\bar{x}_{1}+\left(1-\xi_{1}\right) \bar{v}_{1}\right)=1 .
$$

The equilibrium fractions of susceptible $\bar{x}_{1}$ can be computed by setting equal to 0 the right hand side of (8) so that it can be expressed as function of $\bar{y}_{1}$ as

$$
\bar{x}_{1}=\frac{\left(\mu+\gamma_{1} \bar{y}_{1}\right)(1-p e)}{\mu+R_{0}^{1}\left(\mu+\gamma_{1}\right) \bar{y}_{1}}
$$


Substituting $\bar{v}_{1}=1-\bar{x}_{1}-\bar{y}_{1}$ and (20) into (19) we obtain that $\bar{y}_{1}$ must solve $G\left(\bar{y}_{1}\right)=1$, where

$$
G(y)=R_{0}^{1}\left[\xi_{1} \frac{\left(\mu+\gamma_{1} y\right)(1-p e)}{\mu+R_{0}^{1}\left(\mu+\gamma_{1}\right) y}+\left(1-\xi_{1}\right)(1-y)\right]
$$

Since we assumed $R_{0}^{1}>1$, we obtain

$$
G^{\prime}(y)=-\frac{R_{0}^{1} \xi_{1} \mu(1-p e)\left[R_{0}^{1} \mu+\left(R_{0}^{1}-1\right) \gamma_{1}\right]}{\left[\mu+R_{0}^{1}\left(\mu+\gamma_{1}\right) y\right]^{2}}-R_{0}^{1}\left(1-\xi_{1}\right)<0
$$

and

$$
G(1)=\frac{R_{0}^{1}\left(\mu+\gamma_{1}\right)(1-p e) \xi_{1}}{R_{0}^{1}\left(\mu+\gamma_{1}\right)+\mu}<1
$$

Hence, $G\left(\bar{y}_{1}\right)=1$ has a unique solution in $(0,1)$ if and only if

$$
G(0)>1 \quad \Longleftrightarrow \quad R_{0}^{1}\left((1-p e) \xi_{1}+\left(1-\xi_{1}\right)\right)=R_{0}^{1}\left(1-\xi_{1} p e\right)=R_{p}^{1}>1
$$

We have then proved

Proposition 1. A sufficient and necessary condition for a Subtype-1-Only Equilibrium to exist is $R_{p}^{1}>1$, i.e, pe $<p e_{1}^{D F}$ defined in (17). Moreover, under the assumption $R_{p}^{1}>1$, the Subtype-1-Only Equilibrium is unique.

The equilibrium fraction of vaccinated individuals $\bar{v}_{1}$ can be computed by setting equal to 0 the right hand side of (9) and it can be expressed as function of $\bar{y}_{1}$ as

$$
\bar{v}_{1}=\frac{\left(\mu+\gamma_{1} \bar{y}_{1}\right) p e}{\mu+\left(1-\xi_{1}\right) R_{0}^{1}\left(\mu+\gamma_{1}\right) \bar{y}_{1}}
$$

For future use, we prove the following

Proposition 2. $\bar{y}_{1}$ at the Subtype-1-Only Equilibrium is a decreasing function of pe on $\left[0, p e_{1}^{D F}\right)$. 
Proof. We write explicitly the dependence of $G$ on pe as $G\left(p e, \bar{y}_{1}(p e)\right)=1$. Since by (21) we know that

$$
\frac{\partial G\left(p e, \bar{y}_{1}(p e)\right)}{\partial \bar{y}_{1}}<0
$$

and also

$$
\frac{\partial G\left(p e, \bar{y}_{1}(p e)\right)}{\partial p e}=-\frac{R_{0}^{1} \xi_{1}\left(\mu+\gamma_{1} \bar{y}_{1}\right)}{\mu+R_{0}^{1}\left(\mu+\gamma_{1}\right) \bar{y}_{1}}<0
$$

by the Implicit Function Theorem we obtain

$$
\bar{y}_{1}^{\prime}(p e)=-\frac{\frac{\partial G\left(p e, \bar{y}_{1}\right)}{\partial p e}}{\frac{\partial G\left(p e, \bar{y}_{1}\right)}{\partial \bar{y}_{1}}}<0
$$

thus proving that $\bar{y}_{1}$ is a decreasing function of $p e$.

Completely similar arguments lead us to state that a Subtype-2-Only Equilibrium $\left(\bar{x}_{2}, \bar{v}_{2}, 0, \bar{y}_{2}\right)$ exists and is unique under the necessary and sufficient condition $R_{p}^{2}>1$.

The equilibrium fractions of susceptible and vaccinated individuals at the equilibrium are given by

$$
\bar{x}_{2}=\frac{\left(\mu+\gamma_{2} \bar{y}_{2}\right)(1-p e)}{\mu+R_{0}^{2}\left(\mu+\gamma_{2}\right) \bar{y}_{2}} \quad \bar{v}_{2}=\frac{\left(\mu+\gamma_{2} \bar{y}_{2}\right) p e}{\mu+\left(1-\xi_{2}\right) R_{0}^{2}\left(\mu+\gamma_{2}\right) \bar{y}_{2}}
$$

where $\bar{y}_{2}$ is the unique solution of equation $H\left(\bar{y}_{2}\right)=1$ where

$$
H\left(\bar{y}_{2}\right)=R_{0}^{2}\left[\frac{\left(\mu+\gamma_{2} \bar{y}_{2}\right)(1-p e)}{\mu+R_{0}^{2}\left(\mu+\gamma_{2}\right) \bar{y}_{2}}+\left(1-\xi_{2}\right)\left(1-\bar{y}_{2}\right)\right]
$$

provided that $R_{p}^{2}>1$. 
In terms of pe, we get that the Subtype-2-Only Equilibrium exists for

$$
p e<p e_{2}^{D F} \quad \text { where } \quad p e_{2}^{D F}=\frac{R_{0}^{2}-1}{R_{0}^{2} \xi_{2}} .
$$

Finally, with the same argument used above, it can be proved that $\bar{y}_{2}$ is a decreasing function of $p e$.

\subsubsection{Stability}

We examine now the stability of the Subtype-1-Only Equilibrium. In order to do that, we consider the Jacobian matrix of (14) at the Subtype-1-Only Equilibrium $E_{1}=\left(\bar{N}_{1}, \bar{x}_{1}, \bar{y}_{1}, 0\right)$ and obtain a matrix of the form:

$$
\mathbf{J}\left(\mathbf{E}_{1}\right)=\left(\begin{array}{cc}
E & F \\
0 & R_{0}^{2}\left(\mu+\gamma_{2}\right)\left[\bar{x}_{1}+\left(1-\xi_{2}\right) \bar{v}_{1}\right]-\left(\mu+\gamma_{2}\right)
\end{array}\right)
$$

where $\mathrm{E}$ is the $3 \times 3$ matrix

$\mathbf{E}=\left(\begin{array}{ccc}-\left(\mu+\gamma_{1} \bar{y}_{1}\right) & 0 & -\bar{N} \gamma_{1} \\ \left(\gamma_{1}-R_{0}^{1}\left(\gamma_{1}+\mu\right)\right) \frac{\bar{x}_{1} \bar{y}_{1}}{\bar{N}} & -\left[\mu+R_{0}^{1}\left(\mu+\gamma_{1}\right) \bar{y}_{1}\right] & -\left[R_{0}^{1}\left(\mu+\gamma_{1}\right)-\gamma_{1}\right] \bar{x}_{1} \\ \left(\mu+\gamma_{1} \bar{y}_{1}\right) \frac{\bar{y}_{1}}{\bar{N}} & R_{0}^{1}\left(\mu+\gamma_{1}\right) \xi_{1} \bar{y}_{1} & {\left[\gamma_{1}-R_{0}^{1}\left(\mu+\gamma_{1}\right)\left(1-\xi_{1}\right)\right] \bar{y}_{1}}\end{array}\right)$

We first show the following

Lemma 1. All the eigenvalues of E have negative real part.

The lemma implies that the Subtype-1-Only Equilibrium is always asymptotically stable when it exists $\left(R_{p}^{1}>1\right)$ in absence of individuals infected with subtype 2 , as has been obtained in similar models with one strain and vaccination [13]. Its proof is in the Appendix.

Since $J\left(E_{1}\right)$ is block-triangular, the set of eigenvalues of $J\left(E_{1}\right)$ is given 
by the union of the set of eigenvalues of $\mathrm{E}$ and

$$
\bar{\lambda}_{4}=R_{0}^{2}\left(\mu+\gamma_{2}\right)\left[\bar{x}_{1}+\left(1-\xi_{2}\right) \bar{v}_{1}\right]-\left(\mu+\gamma_{2}\right)
$$

Hence, the Subtype-1-Only Equilibrium is stable for (14) if and only if

$$
\bar{\lambda}_{4}=R_{0}^{2}\left(\mu+\gamma_{2}\right)\left[\bar{x}_{1}+\left(1-\xi_{2}\right) \bar{v}_{1}\right]-\left(\mu+\gamma_{2}\right)<0 .
$$

Rearranging the terms, the Subtype-1-Only Equilibrium is stable if and only if

$$
R_{p}^{2: 1}=R_{0}^{2}\left(\bar{x}_{1}+\left(1-\xi_{2}\right) \bar{v}_{1}\right)<1
$$

We wish now to express (24) in terms of $\bar{y}_{1}$ only. To this aim, one can immediately insert (20) into (24). Instead, to obtain a simpler expression that does not contain pe, we substitute (20) and (22) into (19) and obtain

$$
\frac{R_{0}^{1}\left(\mu+\gamma_{1} \overline{y_{1}}\right)}{\mu+R_{0}^{1}\left(\mu+\gamma_{1}\right) \overline{y_{1}}}\left(1-\frac{p e \mu \xi_{1}}{\mu+R_{0}^{1}\left(\mu+\gamma_{1}\right)\left(1-\xi_{1}\right) \overline{y_{1}}}\right)=1
$$

and by algebraic manipulation of the expression we may write pe as function of $\bar{y}_{1}$

$$
p e=\frac{\left[R_{0}^{1}\left(1-\overline{y_{1}}\right)-1\right]\left[\mu+R_{0}^{1}\left(\mu+\gamma_{1}\right)\left(1-\xi_{1}\right) \overline{y_{1}}\right]}{R_{0}^{1}\left(\mu+\gamma_{1} \overline{y_{1}}\right) \xi_{1}}
$$

Substituting (25) into (20) we obtain the following expression for equilibrium fraction of effectively vaccinated individuals

$$
\bar{v}_{1}=\frac{R_{0}^{1}\left(1-\bar{y}_{1}\right)-1}{R_{0}^{1} \xi_{1}}
$$


Finally we obtain from (24)

$$
\begin{aligned}
R_{p}^{2: 1} & =R_{0}^{2}\left[\bar{x}_{1}+\left(1-\xi_{2}\right) \bar{v}_{1}\right] \\
& =R_{0}^{2}\left[\bar{x}_{1}+\left(1-\xi_{1}\right) \bar{v}_{1}+\left(\xi_{1}-\xi_{2}\right) \bar{v}_{1}\right] \\
& =(\operatorname{using}(19)) R_{0}^{2}\left[\frac{1}{R_{0}^{1}}+\left(\xi_{1}-\xi_{2}\right) \bar{v}_{1}\right] \\
& =\frac{R_{0}^{2}}{R_{0}^{1}}\left[1+\frac{\xi_{1}-\xi_{2}}{\xi_{1}}\left[R_{0}^{1}\left(1-\bar{y}_{1}\right)-1\right]\right] .
\end{aligned}
$$

Summarizing, we have obtained:

Proposition 3. The Subtype-1-Only Equilibrium $E_{1}=\left(\bar{N}_{1}, \bar{x}_{1}, \bar{y}_{1}, 0\right)$ is asymptotically stable [unstable] if $R_{p}^{2: 1}<[>] 1$, where $R_{p}^{2: 1}$ is given by expression (24) or (27).

We now wish to express condition $R_{p}^{2: 1}<1$ in terms of $p e$.

If $\xi_{1} \leq \xi_{2}$, (27) implies that $R_{p}^{2: 1}<1$. In other words, if $\xi_{1} \leq \xi_{2}$, the Subtype-1-Only Equilibrium is asymptotically stable, when it exists.

Therefore, we study the condition $R_{p}^{2: 1}<1$ under the additional assumption $\xi_{1}>\xi_{2}$.

Since $\frac{\left(\xi_{1}-\xi_{2}\right)}{\xi_{1}} R_{0}^{2} \bar{y}_{1}>0$ and $\frac{R_{0}^{2}}{R_{0}^{1}}<1$, expression (27) implies that

$$
\begin{aligned}
R_{p}^{2: 1} & =\frac{R_{0}^{2}}{R_{0}^{1}}\left[1+\frac{\left(\xi_{1}-\xi_{2}\right)}{\xi_{1}} R_{0}^{1}-\frac{\left(\xi_{1}-\xi_{2}\right)}{\xi_{1}}\right]-\frac{\left(\xi_{1}-\xi_{2}\right)}{\xi_{1}} R_{0}^{2} \bar{y}_{1} \\
& <\frac{R_{0}^{2}}{R_{0}^{1}}\left[R_{0}^{1}-\frac{\xi_{2}}{\xi_{1}} R_{0}^{1}+\frac{\xi_{2}}{\xi_{1}}\right] .
\end{aligned}
$$

Recalling the definition (17), we see that

$$
\frac{R_{0}^{2}}{R_{0}^{1}}\left[R_{0}^{1}-\frac{\xi_{2}}{\xi_{1}} R_{0}^{1}+\frac{\xi_{2}}{\xi_{1}}\right] \leq 1 \Longleftrightarrow p e_{2}^{D F} \leq p e_{1}^{D F} ;
$$


hence inequality (28) shows that, if $R_{0}^{1}>R_{0}^{2}, \xi_{1}>\xi_{2}$ and $p e_{2}^{D F} \leq p e_{1}^{D F}$, then $R_{p}^{2: 1}<1$ for every value of $0 \leq p e \leq 1$.

In order to find when $R_{p}^{2: 1}>1$, we then require the assumptions

$$
R_{0}^{1}>R_{0}^{2}, \quad \xi_{1}>\xi_{2} \quad \text { and } \quad p e_{2}^{D F}>p e_{1}^{D F} .
$$

Let us now set $R_{p}^{2: 1}=1$ and find from (27) the corresponding fraction of infected individuals

$$
\bar{y}_{1}^{B P}=\frac{R_{0}^{1} R_{0}^{2}\left(\xi_{1}-\xi_{2}\right)+R_{0}^{2} \xi_{2}-R_{0}^{1} \xi_{1}}{R_{0}^{1} R_{0}^{2}\left(\xi_{1}-\xi_{2}\right)}=\frac{\xi_{1} \xi_{2}}{\xi_{1}-\xi_{2}}\left(p e_{2}^{D F}-p e_{1}^{D F}\right) .
$$

The superscript $B P$ is related to the fact that this value corresponds to a branching point of equilibrium curves, as will be seen later.

Because of the monotonic dependence of $R_{p}^{2: 1}$ on $\bar{y}_{1}(27)$, we have

$$
R_{p}^{2: 1}<[>] 1 \quad \Longleftrightarrow \quad \bar{y}_{1}>[<] \bar{y}_{1}^{B P}
$$

By (25) and (30) we can compute the pe values at which branching occurs. We see that $R_{p}^{2: 1}=1$ for

$p e_{1}^{B P}=\hat{v} \frac{R_{0}^{1} R_{0}^{2}\left(\xi_{1}-\xi_{2}\right)\left[R_{0}^{1}\left(\mu+\gamma_{1}\right)\left(1-\xi_{1}\right)+\mu\right]+R_{0}^{1}\left(\mu+\gamma_{1}\right)\left(1-\xi_{1}\right)\left(R_{0}^{2} \xi_{2}-R_{0}^{1} \xi_{1}\right)}{\left[R_{0}^{1} R_{0}^{2}\left(\xi_{1}-\xi_{2}\right)\left(\mu+\gamma_{1}\right)+\gamma_{1}\left(R_{0}^{2} \xi_{2}-R_{0}^{1} \xi_{1}\right)\right]}$

where

$$
\hat{v}=\frac{\left(R_{0}^{1}-R_{0}^{2}\right)}{R_{0}^{1} R_{0}^{2}\left(\xi_{1}-\xi_{2}\right)}
$$

Since $\bar{y}_{1}$ is a decreasing function of pe (see Proposition 2), we conclude that

$$
R_{p}^{2: 1}<[>] 1 \Longleftrightarrow p e<[>] p e_{1}^{B P}
$$


By algebraic manipulation of (31), $p e_{1}^{B P}$ may be written as

$$
p e_{1}^{B P}=\frac{\left(R_{0}^{1}-R_{0}^{2}\right)}{R_{0}^{2}\left(\xi_{1}-\xi_{2}\right)}\left[1-\xi_{1}+\frac{\mu \xi_{1}\left(R_{0}^{2}\left(1-\xi_{2}\right)-R_{0}^{1}\left(1-\xi_{1}\right)\right)}{\left(C \gamma_{1}+\mu R_{0}^{1} R_{0}^{2}\left(\xi_{1}-\xi_{2}\right)\right)}\right]
$$

where

$$
C=R_{0}^{1} R_{0}^{2}\left(\xi_{1}-\xi_{2}\right)+R_{0}^{2} \xi_{2}-R_{0}^{1} \xi_{1}
$$

It is easy to see that

$$
C>0 \Longleftrightarrow p e_{2}^{D F}>p e_{1}^{D F}
$$

To proceed, we use the following

Lemma 2. Assume (29).

a) If $R_{0}^{2}\left(1-\xi_{2}\right)>R_{0}^{1}\left(1-\xi_{1}\right)$, then $0<p e_{1}^{B P}<1$ at least for $\mu / \gamma_{1}>0$ small enough.

b) If $R_{0}^{2}\left(1-\xi_{2}\right) \leq R_{0}^{1}\left(1-\xi_{1}\right)$, then $p e_{1}^{B P} \geq 1$ for all $\mu>0$.

The proof is in the Appendix.

The assumption $R_{0}^{2}\left(1-\xi_{2}\right)>R_{0}^{1}\left(1-\xi_{1}\right)$ is then necessary for strain 2 to be able to invade the Subtype-1-Only Equilibrium.

Summing up, necessary assumptions for having $R_{p}^{2: 1}>1$ with $p e \leq 1$ are

$$
\left\{\begin{array}{l}
\xi_{1}>\xi_{2} \\
p e_{2}^{D F}>p e_{1}^{D F} \\
R_{0}^{2}\left(1-\xi_{2}\right)>R_{0}^{1}\left(1-\xi_{1}\right)
\end{array}\right.
$$

We conclude the following 
Proposition 4. Under the assumption $R_{0}^{1}>R_{0}^{2}$, if any of the conditions (36) is violated, then the Subtype-1-Only Equilibrium is asymptotically stable for all $0 \leq p e \leq 1$ in which this equilibrium is defined. If all of (36) are satisfied, then the Subtype-1-Only Equilibrium (when it is defined) is asymptotically stable for $0 \leq p e \leq p e_{1}^{B P}$ and unstable for $p e>p e_{1}^{B P}$, where $p e_{1}^{B P}$ is defined by (31) or (33). Under (36) the quantity $p e_{1}^{B P}<1$ at least for $\mu$ small enough.

Completely similar (but reversed) arguments apply to the Subtype-2Only Equilibrium. It is asymptotically stable [unstable] if

$$
R_{p}^{1: 2}=R_{0}^{1}\left(\bar{x}_{2}+\left(1-\xi_{1}\right) \bar{v}_{2}\right)<[>] 1
$$

As before, we may write $R_{p}^{1: 2}$ as

$$
R_{p}^{1: 2}=\frac{R_{0}^{1}}{R_{0}^{2}}\left[1+\frac{\xi_{2}-\xi_{1}}{\xi_{2}}\left[R_{0}^{2}\left(1-\bar{y}_{2}\right)-1\right]\right]
$$

Again, if we assume that $R_{0}^{1}>R_{0}^{2}$, then (37) together with

$$
\bar{v}_{2}=\frac{R_{0}^{2}\left(1-\bar{y}_{2}\right)-1}{R_{0}^{2} \xi_{1}}>0
$$

implies that

$$
\xi_{2} \geq \xi_{1} \Longrightarrow R_{p}^{1: 2}>1
$$

That is, if $R_{0}^{1}>R_{0}^{2}$ and $\xi_{2} \geq \xi_{1}$, then Subtype-1 invades the Subtype-2-Only Equilibrium, wherever it exists.

Assume now $\xi_{2}<\xi_{1}$ together with $R_{0}^{1}>R_{0}^{2}$. By the same reasoning made before, expression (37) implies

$$
R_{p}^{1: 2}=\frac{R_{0}^{1}}{R_{0}^{2}}\left[1+\frac{\xi_{2}-\xi_{1}}{\xi_{2}}\left(R_{0}^{2}-1\right)\right]+\frac{\xi_{1}-\xi_{2}}{\xi_{2}} \bar{y}_{2}>\frac{R_{0}^{1}}{R_{0}^{2}}\left[R_{0}^{2}-\frac{\xi_{1}}{\xi_{2}} R_{0}^{2}+\frac{\xi_{1}}{\xi_{2}}\right]
$$


The right hand side of (39) is greater or equal than 1 , if and only if $C \leq 0$ with $C$ defined in (36) (alternatively, see Table 2). Hence

$$
C \leq 0 \Longrightarrow R_{p}^{1: 2}>1
$$

i.e. strain 1 invades the Subtype-2-Only Equilibrium whenever this exists. To proceed, we also assume $C>0$.

As before, we find the fraction of individuals infected with strain 2 at the equilibrium corresponding to $R_{p}^{1: 2}=1$ :

$$
\bar{y}_{2}^{B P}=\frac{R_{0}^{1} R_{0}^{2}\left(\xi_{1}-\xi_{2}\right)+R_{0}^{2} \xi_{2}-R_{0}^{1} \xi_{1}}{R_{0}^{1} R_{0}^{2}\left(\xi_{1}-\xi_{2}\right)}=\frac{C}{R_{0}^{1} R_{0}^{2}\left(\xi_{1}-\xi_{2}\right)}
$$

Notice that, since (37) is an increasing function of $\bar{y}_{2}$ (remember $\xi_{1}>\xi_{2}$ ), we have

$$
R_{p}^{1: 2}<1 \quad \Longleftrightarrow \quad \bar{y}_{2}<\bar{y}_{2}^{B P}
$$

Writing, analogously to (25), pe as function of $\bar{y}_{2}$ as

$$
p e=\frac{\left[R_{0}^{2}\left(1-\bar{y}_{2}\right)-1\right]\left[\mu+R_{0}^{2}\left(\mu+\gamma_{2}\right)\left(1-\xi_{2}\right) \bar{y}_{2}\right]}{R_{0}^{2} \xi_{2}\left(\mu+\gamma_{2} \bar{y}_{2}\right)}
$$

we see that $R_{p}^{1: 2}=1$ for

$p e_{2}^{B P}=\hat{v} \frac{R_{0}^{1} R_{0}^{2}\left(\xi_{1}-\xi_{2}\right)\left[R_{0}^{2}\left(\mu+\gamma_{2}\right)\left(1-\xi_{2}\right)+\mu\right]+R_{0}^{2}\left(R_{0}^{2} \xi_{2}-R_{0}^{1} \xi_{1}\right)\left(\mu+\gamma_{2}\right)\left(1-\xi_{2}\right)}{\left[R_{0}^{1} R_{0}^{2}\left(\xi_{1}-\xi_{2}\right)\left(\mu+\gamma_{2}\right)+\gamma_{2}\left(R_{0}^{2} \xi_{2}-R_{0}^{1} \xi_{1}\right)\right]}$

using the definition (32) for $\hat{v}$.

Since $\bar{y}_{2}$ is a decreasing function of pe (by the same argument that led to Proposition 2), we conclude that

$$
R_{p}^{1: 2}<1 \quad \Longleftrightarrow p e>p e_{2}^{B P}
$$


By manipulation of (41) we find that

$$
p e_{2}^{B P}=\frac{\left(R_{0}^{1}-R_{0}^{2}\right)}{R_{0}^{1}\left(\xi_{1}-\xi_{2}\right)}\left[1-\xi_{2}+\frac{\mu \xi_{2}\left(R_{0}^{2}\left(1-\xi_{2}\right)-R_{0}^{1}\left(1-\xi_{1}\right)\right)}{\gamma_{2} C+\mu R_{0}^{1} R_{0}^{2}\left(\xi_{1}-\xi_{2}\right)}\right] .
$$

Anagolously to Lemma 2, we have

Lemma 3. Assume (29).

a) If $R_{0}^{2}\left(1-\xi_{2}\right)>R_{0}^{1}\left(1-\xi_{1}\right)$, then $0<p e_{2}^{B P}<1$ at least for $\mu / \gamma_{2}>0$ small enough.

b) If $R_{0}^{2}\left(1-\xi_{2}\right) \leq R_{0}^{1}\left(1-\xi_{1}\right)$, then $p e_{2}^{B P} \geq 1$ for all $\mu>0$.

The proof is identical to that of Lemma 2 and is skipped.

Symmetrically to Proposition 4, we obtain

Proposition 5. Under the assumption $R_{0}^{1}>R_{0}^{2}$, if any of the conditions (36) is violated, then the Subtype-2-Only Equilibrium is unstable for all $0 \leq$ pe $\leq 1$ in which this equilibrium is defined. If all of (36) are satisfied,

then the Subtype-2-Only Equilibrium is unstable for $0 \leq p e \leq p e_{2}^{B P}$ and asymptotically stable for pe $>p e_{2}^{B P}$ (when the equilibrium itself is defined), where $p e_{2}^{B P}$ is defined by (41) or (42). Under (36) the quantity $p e_{2}^{B P}<1$ at least for $\mu$ small enough.

\subsection{Coexistence Equilibrium}

\subsubsection{Existence}

At the Coexistence Equilibrium completely susceptible $(\hat{x}>0)$, effectively vaccinated $(\hat{v}>0)$, individuals infected by subtype $1\left(\hat{y}_{1}>0\right)$ and individuals infected by subtype $2\left(\hat{y}_{2}>0\right)$ are all present in the community. 
Setting equal to 0 equations (10)-(11), together with (15) one obtains

$$
\left\{\begin{array}{l}
R_{0}^{1}\left[\hat{x}+\left(1-\xi_{1}\right) \hat{v}\right]=1 \\
R_{0}^{2}\left[\hat{x}+\left(1-\xi_{2}\right) \hat{v}\right]=1
\end{array}\right.
$$

The equilibrium fractions $\hat{x}, \hat{v}$ can be computed solving (43):

$$
\hat{x}=\frac{R_{0}^{2}\left(1-\xi_{2}\right)-R_{0}^{1}\left(1-\xi_{1}\right)}{R_{0}^{1} R_{0}^{2}\left(\xi_{1}-\xi_{2}\right)} \quad \hat{v}=\frac{R_{0}^{1}-R_{0}^{2}}{R_{0}^{1} R_{0}^{2}\left(\xi_{1}-\xi_{2}\right)}
$$

Fraction $\hat{v}$ is positive under the condition that

$$
\text { if } R_{0}^{i}>R_{0}^{j} \text {, then } \xi_{i}>\xi_{j}
$$

which means that the Coexistence Equilibrium exists only if the vaccine induces a higher degree of protection against the subtype with the higher fitness in a completely susceptible population.

Without loss of generality, let's assume $R_{0}^{1}>R_{0}^{2}$ and require $\xi_{1}>\xi_{2}$.

The susceptible fraction $\hat{x}$ is positive if and only if

$$
R_{0}^{2}\left(1-\xi_{2}\right)>R_{0}^{1}\left(1-\xi_{1}\right)
$$

By substitution of (44) into $\hat{x}+\hat{v}<1$ one obtains

$$
\frac{R_{0}^{1} \xi_{1}-R_{0}^{2} \xi_{2}}{R_{0}^{1} R_{0}^{2}\left(\xi_{1}-\xi_{2}\right)}<1 \quad \Longleftrightarrow C>0
$$

where $C$ is given by (34).

Conditions (36) are then necessary for the existence of a Coexistence Equilibrium. 
Setting equal to 0 equations (8)-(9) together with (15) and using matrix notation, one obtains

$$
A\left(\begin{array}{l}
\hat{y}_{1} \\
\hat{y}_{2}
\end{array}\right)=\mu\left(\begin{array}{c}
1-p e-\hat{x} \\
p e-\hat{v}
\end{array}\right)
$$

where

$$
A=\left(\begin{array}{cc}
R_{0}^{1}\left(\mu+\gamma_{1}\right) \hat{x}-\gamma_{1}(1-p e) & R_{0}^{2}\left(\mu+\gamma_{2}\right) \hat{x}-\gamma_{2}(1-p e) \\
R_{0}^{1}\left(\mu+\gamma_{1}\right)\left(1-\xi_{1}\right) \hat{v}-\gamma_{1} p e & R_{0}^{2}\left(\mu+\gamma_{2}\right)\left(1-\xi_{2}\right) \hat{v}-\gamma_{2} p e
\end{array}\right)
$$

System (45) admits a unique solution if and only if

$$
|A|=\mu\left[R_{0}^{2}\left(\mu+\gamma_{2}\right)\left(1-\xi_{2}\right) \hat{v}-R_{0}^{1}\left(\mu+\gamma_{1}\right)\left(1-\xi_{1}\right) \hat{v}+p e\left(\gamma_{1}-\gamma_{2}\right)\right] \neq 0
$$

Observation 1. If $\gamma_{1}=\gamma_{2}$, then by (36) $|A|>0$ for $0 \leq p e \leq 1$.

Under the alternative assumption that $|A| \neq 0$, we can explicitly solve 
(45) by Cramer's rule

$$
\begin{aligned}
\hat{y}_{1}= & \frac{\left|\begin{array}{cc}
\mu(1-p e-\hat{x}) & R_{0}^{2}\left(\mu+\gamma_{2}\right) \hat{x}-\gamma_{2}(1-p e) \\
\mu(p e-\hat{v}) & R_{0}^{2}\left(\mu+\gamma_{2}\right)\left(1-\xi_{2}\right) \hat{v}-\gamma_{2} p e
\end{array}\right|}{|A|} \\
= & \frac{p e \mu\left[\gamma_{2}(\hat{x}+\hat{v})-\left(\mu+\gamma_{2}\right)\right]+\mu \hat{v}\left[R_{0}^{2}\left(\mu+\gamma_{2}\right)\left(1-\xi_{2}+\xi_{2} \hat{x}\right)-\gamma_{2}\right]}{|A|} \\
\hat{y}_{2}= & \frac{\left|\begin{array}{cc}
R_{0}^{1}\left(\mu+\gamma_{1}\right) \hat{x}-\gamma_{1}(1-p e) \\
R_{0}^{1}\left(\mu+\gamma_{1}\right)\left(1-\xi_{1}\right) \hat{v}-\gamma_{1} p e
\end{array}\right|}{\mid A(p e-\hat{v})} \mid \\
= & \frac{p e \mu\left[\gamma_{1}(1-(\hat{x}+\hat{v}))+\mu\right]+\mu \hat{v}\left[\gamma_{1}-R_{0}^{1}\left(\mu+\gamma_{1}\right)\left(1-\xi_{1}+\xi_{1} \hat{x}\right)\right]}{|A|}
\end{aligned}
$$

We conclude the following

Proposition 6. Under the assumption $R_{0}^{1}>R_{0}^{2}$, necessary conditions for a Coexistence Equilibrium to exist are given by (36). Moreover, if $\gamma_{1}=\gamma_{2}$ the Coexistence Equilibrium is unique. If $\gamma_{1} \neq \gamma_{2}$ the Coexistence Equilibrium is unique under the assumption that $|A| \neq 0$, where $|A|$ is given by (46).

In order to find sufficient conditions for the existence of a positive equilibrium, we start with the assumption $|A|>0$.

By (48) one obtains that $\hat{y}_{2}>0$ for

$$
p e>\frac{\hat{v}\left[R_{0}^{1}\left(\mu+\gamma_{1}\right)\left(1-\xi_{1}+\xi_{1} \hat{x}\right)-\gamma_{1}\right]}{\mu+\gamma_{1}(1-(\hat{x}+\hat{v}))}
$$


Substituting (44) and rearranging the terms one gets

$$
\begin{aligned}
& p e>\hat{v} \frac{\left[R_{0}^{1}\left(\mu+\gamma_{1}\right)\left(1-\xi_{1}\right)+R_{0}^{1} \xi_{1}\left(\mu+\gamma_{1}\right) \frac{R_{0}^{2}\left(1-\xi_{2}\right)-R_{0}^{1}\left(1-\xi_{1}\right)}{R_{0}^{1} R_{0}^{2}\left(\xi_{1}-\xi_{2}\right)}-\gamma_{1}\right]}{R_{0}^{1} R_{0}^{2}\left(\xi_{1}-\xi_{2}\right) \mu+\gamma_{1}\left[R_{0}^{1} R_{0}^{2}\left(\xi_{1}-\xi_{2}\right)-R_{0}^{1} \xi_{1}+R_{0}^{2} \xi_{2}\right]} \\
& p e>\hat{v} \frac{R_{0}^{1} R_{0}^{2}\left(\xi_{1}-\xi_{2}\right)\left[R_{0}^{1}\left(\mu+\gamma_{1}\right)\left(1-\xi_{1}\right)-\gamma_{1}\right]+R_{0}^{1} \xi_{1}\left(\mu+\gamma_{1}\right)\left[R_{0}^{2}\left(1-\xi_{2}\right)-R_{0}^{1}\left(1-\xi_{1}\right)\right]}{R_{0}^{1} R_{0}^{2}\left(\xi_{1}-\xi_{2}\right)\left(\mu+\gamma_{1}\right)+\gamma_{1}\left(R_{0}^{2} \xi_{2}-R_{0}^{1} \xi_{1}\right)} \\
& p e>\hat{v} \frac{R_{0}^{1} R_{0}^{2}\left(\xi_{1}-\xi_{2}\right)\left[R_{0}^{1}\left(\mu+\gamma_{1}\right)\left(1-\xi_{1}\right)+\mu\right]+R_{0}^{1}\left(\mu+\gamma_{1}\right)\left(1-\xi_{1}\right)\left(R_{0}^{2} \xi_{2}-R_{0}^{1} \xi_{1}\right)}{R_{0}^{1} R_{0}^{2}\left(\xi_{1}-\xi_{2}\right)\left(\mu+\gamma_{1}\right)+\gamma_{1}\left(R_{0}^{2} \xi_{2}-R_{0}^{1} \xi_{1}\right)}
\end{aligned}
$$

By (31) inequality (49) can be written as

$$
p e>p e_{1}^{B P}
$$

Similarly, by (47), condition $\hat{y}_{1}>0$ can be expressed in terms of $p e$

$$
p e<\frac{\hat{x} \hat{v}\left[R_{0}^{2} \xi_{2}\left(\mu+\gamma_{2}\right)\right]+\hat{v}\left[R_{0}^{2}\left(\mu+\gamma_{2}\right)\left(1-\xi_{2}\right)-\gamma_{2}\right]}{\mu+\gamma_{2}(1-(\hat{x}+\hat{v}))}
$$

Substituting (44) and rearranging the terms one gets

$$
\begin{aligned}
p e & <\hat{v} \frac{\left[R_{0}^{2}\left(1-\xi_{2}\right)-R_{0}^{1}\left(1-\xi_{1}\right)\right]\left[R_{0}^{2} \xi_{2}\left(\mu+\gamma_{2}\right)\right]+\left[R_{0}^{2}\left(\mu+\gamma_{2}\right)\left(1-\xi_{2}\right)-\gamma_{2}\right]}{\left[R_{0}^{1} R_{0}^{2}\left(\xi_{1}-\xi_{2}\right)\left(\mu+\gamma_{2}\right)+\gamma_{2}\left(R_{0}^{1} \xi_{1}-R_{0}^{2} \xi_{2}\right)\right]} \\
p e & <\hat{v} \frac{R_{0}^{1} R_{0}^{2}\left(\xi_{1}-\xi_{2}\right)\left[R_{0}^{2}\left(\mu+\gamma_{2}\right)\left(1-\xi_{2}\right)-\gamma_{2}\right]+R_{0}^{2} \xi_{2}\left(\mu+\gamma_{2}\right)\left[R_{0}^{2}\left(1-\xi_{2}\right)-R_{0}^{1}\left(1-\xi_{1}\right)\right]}{R_{0}^{1} R_{0}^{2}\left(\xi_{1}-\xi_{2}\right)\left(\mu+\gamma_{2}\right)+\gamma_{2}\left(R_{0}^{2} \xi_{2}-R_{0}^{1} \xi_{1}\right)} \\
p e & <\hat{v} \frac{R_{0}^{1} R_{0}^{2}\left(\xi_{1}-\xi_{2}\right)\left[R_{0}^{2}\left(\mu+\gamma_{2}\right)\left(1-\xi_{2}\right)+\mu\right]+R_{0}^{2}\left(R_{0}^{2} \xi_{2}-R_{0}^{1} \xi_{1}\right)\left(\mu+\gamma_{2}\right)\left(1-\xi_{2}\right)}{\left[R_{0}^{1} R_{0}^{2}\left(\xi_{1}-\xi_{2}\right)\left(\mu+\gamma_{2}\right)+\gamma_{2}\left(R_{0}^{2} \xi_{2}-R_{0}^{1} \xi_{1}\right)\right]}
\end{aligned}
$$

By (41) inequality (50) can be written as

$$
p e<p e_{2}^{B P}
$$

With similar (but reversed) arguments, one finds that under the assumption $|A|<0, \hat{y}_{2}>0$ and $\hat{y}_{1}>0$ for

$$
p e_{2}^{B P}<p e<p e_{1}^{B P}
$$


We have then proved the following

Proposition 7. Under the assumptions $R_{0}^{1}>R_{0}^{2}$ and (36), sufficient and necessary conditions for the Coexistence Equilibrium to exist are

(a) if $|A|>0, R_{p}^{1: 2}>1$ and $R_{p}^{2: 1}>1$ (i.e. $p e_{1}^{B P}<p e<p e_{2}^{B P}$ );

(b) if $|A|<0, R_{p}^{1: 2}<1$ and $R_{p}^{2: 1}<1$ (i.e. $p e_{2}^{B P}<p e<p e_{1}^{B P}$ ).

where $p e_{1}^{B P}$ and $p e_{2}^{B P}$ are given by (33) and (42) respectively.

\subsubsection{Conditions for sub- or super-critical bifurcations}

It is therefore relevant finding whether $p e_{1}^{B P}<p e_{2}^{B P}$ or vice versa.

Lemma 4. Under the assumption $R_{0}^{1}>R_{0}^{2}$ and (36), $p e_{1}^{B P}$ and $p e_{2}^{B P}$, given by (33) and (42), are decreasing functions of $\gamma_{1}$ and $\gamma_{2}$ respectively. If $\gamma_{1} \geq \gamma_{2}$ then $p e_{1}^{B P}<p e_{2}^{B P}$.

If

$$
R_{0}^{2}\left(\xi_{1}-\xi_{2}\right) \geq \xi_{1}
$$

then $p e_{1}^{B P}<p e_{2}^{B P}$ for all values of $\gamma_{1}$ and $\gamma_{2}$.

If $R_{0}^{2}\left(\xi_{1}-\xi_{2}\right)<\xi_{1}$ then $p e_{1}^{B P}>p e_{2}^{B P}$ for $\gamma_{1}$ small enough, and $\gamma_{2}$ large enough.

Proof. The fact that $p e_{1}^{B P}$ and $p e_{2}^{B P}$ are decreasing functions of $\gamma_{1}$ and $\gamma_{2}$ is an immediate consequence of expressions (33) and (42) and assumptions (36).

Consider now $\gamma_{1}=\gamma_{2}=\gamma$. With some simple algebraic manipulations, 
by substitution of equation (33) for $p e_{1}^{B P}$ and (42) for $p e_{2}^{B P}$ one obtains

$$
\begin{gathered}
p e_{2}^{B P}-p e_{1}^{B P}=\frac{\left(R_{0}^{1}-R_{0}^{2}\right)\left(1-\xi_{2}\right)}{R_{0}^{2}\left(\xi_{1}-\xi_{2}\right)}-\frac{\left(R_{0}^{1}-R_{0}^{2}\right)\left(1-\xi_{1}\right)}{R_{0}^{2}\left(\xi_{1}-\xi_{2}\right)} \\
+\frac{\mu\left(R_{0}^{2}\left(1-\xi_{2}\right)-R_{0}^{1}\left(1-\xi_{1}\right)\right)}{\left(C \gamma+\mu R_{0}^{1} R_{0}^{2}\left(\xi_{1}-\xi_{2}\right)\right)}\left[\frac{\left(R_{0}^{1}-R_{0}^{2}\right) \xi_{2}}{R_{0}^{2}\left(\xi_{1}-\xi_{2}\right)}-\frac{\left(R_{0}^{1}-R_{0}^{2}\right) \xi_{1}}{R_{0}^{2}\left(\xi_{1}-\xi_{2}\right)}\right] \\
=\frac{\left(R_{0}^{1}-R_{0}^{2}\right)\left(R_{0}^{2}\left(1-\xi_{2}\right)-R_{0}^{1}\left(1-\xi_{1}\right)\right)(\mu+\gamma) C}{R_{0}^{1} R_{0}^{2}\left(\xi_{1}-\xi_{2}\right)\left(C \gamma+\mu R_{0}^{1} R_{0}^{2}\left(\xi_{1}-\xi_{2}\right)\right)}>0 .
\end{gathered}
$$

From the fact that $p e_{1}^{B P}$ and $p e_{2}^{B P}$ are decreasing functions of $\gamma_{1}$ and $\gamma_{2}$ respectively, we may conclude that the inequality $p e_{1}^{B P}<p e_{2}^{B P}$ holds also for every $\gamma_{1}>\gamma_{2}$.

As for the final claim, if $\lim _{\gamma_{1} \rightarrow 0} p e_{1}^{B P} \leq \lim _{\gamma_{2} \rightarrow+\infty} p e_{2}^{B P}$, then $p e_{1}^{B P}<p e_{2}^{B P}$ for all finite, positive $\gamma_{1}$ and $\gamma_{2}$.

Vice versa, if $\lim _{\gamma_{1} \rightarrow 0} p e_{1}^{B P}>\lim _{\gamma_{2} \rightarrow+\infty} p e_{2}^{B P}$, by continuity $p e_{1}^{B P}>p e_{2}^{B P}$ over some range of $\gamma_{1}$ and $\gamma_{2}$ values.

$$
\lim _{\gamma_{1} \rightarrow 0} p e_{1}^{B P}=\frac{\left(R_{0}^{1}-R_{0}^{2}\right)}{R_{0}^{2}\left(\xi_{1}-\xi_{2}\right)}\left[1-\xi_{1}+\frac{\xi_{1}\left(R_{0}^{2}\left(1-\xi_{2}\right)-R_{0}^{1}\left(1-\xi_{1}\right)\right)}{R_{0}^{1} R_{0}^{2}\left(\xi_{1}-\xi_{2}\right)}\right]
$$

while

$$
\lim _{\gamma_{2} \rightarrow+\infty} p e_{2}^{B P}=\frac{\left(R_{0}^{1}-R_{0}^{2}\right)\left(1-\xi_{2}\right)}{R_{0}^{2}\left(\xi_{1}-\xi_{2}\right)}
$$

Hence, with some algebra

$$
\begin{aligned}
\lim _{\gamma_{1} \rightarrow 0} p e_{1}^{B P} & -\lim _{\gamma_{2} \rightarrow+\infty} p e_{2}^{B P} \\
& =\frac{\left(R_{0}^{2}\left(1-\xi_{2}\right)-R_{0}^{1}\left(1-\xi_{1}\right)\right)\left(R_{0}^{1}-R_{0}^{2}\right)}{R_{0}^{1} R_{0}^{2}\left(\xi_{1}-\xi_{2}\right)}\left(-1+\frac{\xi_{1}}{R_{0}^{2}\left(\xi_{1}-\xi_{2}\right)}\right) .
\end{aligned}
$$

This quantity is positive if and only if $R_{0}^{2}\left(\xi_{1}-\xi_{2}\right)<\xi_{1}$, yielding the conclusion of the proof.

Finally, we show 
Lemma 5. Under the assumptions $R_{0}^{1}>R_{0}^{2}$ and (36), the following implications hold:

(a) if $p e_{1}^{B P}<p e_{2}^{B P}$, then $|A|>0$ for all $p e \in\left[p e_{1}^{B P}, p e_{2}^{B P}\right]$;

(b) if $p e_{2}^{B P}<p e_{1}^{B P}$, then $|A|<0$ for all $p e \in\left[p e_{2}^{B P}, p e_{1}^{B P}\right]$.

(c) if $p e_{2}^{B P}=p e_{1}^{B P}$, then $|A|=0$ for $p e=p e_{1}^{B P}=p e_{2}^{B P}$.

where $p e_{1}^{B P}$ is given in (33) and $p e_{2}^{B P}$ is given in (42) (alternatively, see Table 1).

Through a) and b), we will be able to draw a clear bifurcation pattern (see for example [15]) of the system, with transcritical bifurcations occurring at $E_{1}$ for $p e=p e_{1}^{B P}$, and at $E_{2}$ for $p e=p e_{2}^{B P}$.

Proof. We denote by $|A|_{B P i}$ the determinant of $A$ computed with $p e=p e_{i}^{B P}$, $i=1,2$. Through simple, but tedious, algebraic manipulations, one arrives at

$$
\begin{aligned}
|A|_{B P 1}= & \frac{\mu\left(R_{0}^{2}\left(1-\xi_{2}\right)-R_{0}^{1}\left(1-\xi_{1}\right)\right)\left(R_{0}^{1}-R_{0}^{2}\right)}{R_{0}^{1} R_{0}^{2}\left(\xi_{1}-\xi_{2}\right)} \\
& \times\left[\mu+\gamma_{2}-\frac{\gamma_{2} \mu R_{0}^{1} \xi_{1}}{C \gamma_{1}+\mu R_{0}^{1} R_{0}^{2}\left(\xi_{1}-\xi_{2}\right)}+\frac{\gamma_{1} \mu R_{0}^{1} \xi_{1}}{C \gamma_{1}+\mu R_{0}^{1} R_{0}^{2}\left(\xi_{1}-\xi_{2}\right)}\right] .
\end{aligned}
$$

Through the computation of the derivative of $|A|_{B P 1}$, it is immediate to see that $|A|_{B P 1}$ is an increasing function of $\gamma_{1}$. We already know (Observation 1) that $|A|_{B P 1}>0$ for $\gamma_{1} \geq \gamma_{2}$.

Setting (52) equal to 0 , we see that

$$
|A|_{B P 1}=0 \Longleftrightarrow \gamma_{1}=\Psi_{1}\left(\gamma_{2}\right)
$$


with

$$
\Psi_{1}\left(\gamma_{2}\right)=\frac{\mu\left(\gamma_{2} R_{0}^{1}\left(\xi_{1}-R_{0}^{2}\left(\xi_{1}-\xi_{2}\right)\right)-\mu R_{0}^{1} R_{0}^{2}\left(\xi_{1}-\xi_{2}\right)\right)}{C\left(\mu+\gamma_{2}\right)+\mu R_{0}^{1} \xi_{1}} .
$$

If $\Psi_{1}\left(\gamma_{2}\right)<0$, then $|A|_{B P 1}>0$ for all $\gamma_{1}>0$. In particular $\Psi_{1}\left(\gamma_{2}\right)<0$ for all $\gamma_{2}>0$ if $R_{0}^{2}\left(\xi_{1}-\xi_{2}\right) \geq \xi_{1}$, i.e. (51) holds.

Otherwise, $|A|_{B P 1}>0$ for $\gamma_{1}>\Psi_{1}\left(\gamma_{2}\right)$ and $|A|_{B P 1}<0$ for $\gamma_{1}<\Psi_{1}\left(\gamma_{2}\right)$.

Similarly, we obtain

$$
\begin{aligned}
|A|_{B P 2}= & \frac{\mu\left(R_{0}^{2}\left(1-\xi_{2}\right)-R_{0}^{1}\left(1-\xi_{1}\right)\right)\left(R_{0}^{1}-R_{0}^{2}\right)}{R_{0}^{1} R_{0}^{2}\left(\xi_{1}-\xi_{2}\right)} \\
& \times\left[\mu-\frac{\gamma_{2} \mu R_{0}^{2} \xi_{2}}{C \gamma_{2}+\mu R_{0}^{1} R_{0}^{2}\left(\xi_{1}-\xi_{2}\right)}+\gamma_{1}+\frac{\gamma_{1} \mu R_{0}^{2} \xi_{2}}{C \gamma_{2}+\mu R_{0}^{1} R_{0}^{2}\left(\xi_{1}-\xi_{2}\right)}\right] .
\end{aligned}
$$

Again, through the computation of the derivative of $|A|_{B P 2}$ it is immediate to see that $|A|_{B P 2}$ is a decreasing function of $\gamma_{2}$ and

$$
\lim _{\gamma_{2} \rightarrow \infty}|A|_{B P 2}=\frac{\mu\left(R_{0}^{2}\left(1-\xi_{2}\right)-R_{0}^{1}\left(1-\xi_{1}\right)\right)\left(R_{0}^{1}-R_{0}^{2}\right)}{R_{0}^{1} R_{0}^{2}\left(\xi_{1}-\xi_{2}\right)}\left(\mu+\gamma_{1}-\frac{\mu R_{0}^{2} \xi_{2}}{C}\right) .
$$

Hence, if

$$
C\left(\mu+\gamma_{1}\right) \geq \mu R_{0}^{2} \xi_{2}
$$

$|A|_{B P 2}>0$ for all $\gamma_{2}>0$.

Otherwise, when (56) does not hold, setting (55) equal to 0, we see that

$$
|A|_{B P 2}=0 \Longleftrightarrow \gamma_{2}=\Psi_{2}\left(\gamma_{1}\right)
$$

with

$$
\Psi_{2}\left(\gamma_{1}\right)=\frac{\mu\left(\gamma_{1} R_{0}^{2}\left(\xi_{2}+R_{0}^{1}\left(\xi_{1}-\xi_{2}\right)\right)+\mu R_{0}^{1} R_{0}^{2}\left(\xi_{1}-\xi_{2}\right)\right)}{\mu R_{0}^{2} \xi_{2}-C\left(\mu+\gamma_{1}\right)}
$$

We see that, if (56) does not hold, $\Psi_{2}\left(\gamma_{1}\right)>0$, and $|A|_{B P 2}>0$ for $\gamma_{2}<\Psi_{2}\left(\gamma_{1}\right)$ and $|A|_{B P 2}<0$ for $\gamma_{2}>\Psi_{2}\left(\gamma_{1}\right)$. 
Finally, we observe that the conditions for $|A|_{B P i}=0, i=1,2$, are actually the same; more precisely $\Psi_{2}\left(\gamma_{1}\right)$ is the inverse of $\Psi_{1}$, defined on the appropriate domain. Indeed, solving the equation $\gamma_{1}=\Psi_{1}\left(\gamma_{2}\right)$ for $\gamma_{2}$, we obtain

$$
\gamma_{2}=\frac{\mu\left(\gamma_{1}\left(C+R_{0}^{1} \xi_{1}\right)+\mu R_{0}^{1} R_{0}^{2}\left(\xi_{1}-\xi_{2}\right)\right)}{\mu R_{0}^{1}\left(\xi_{1}-R_{0}^{2}\left(\xi_{1}-\xi_{2}\right)\right)-C \gamma_{1}}=\Psi_{2}\left(\gamma_{1}\right)
$$

where the last identity comes from the definition of $C$, so that

$C+R_{0}^{1} \xi_{1}=R_{0}^{2}\left(\xi_{2}+R_{0}^{1}\left(\xi_{1}-\xi_{2}\right)\right) \quad$ and $\quad R_{0}^{1}\left(\xi_{1}-R_{0}^{2}\left(\xi_{1}-\xi_{2}\right)\right)=-C+R_{0}^{2} \xi_{2}$.

Summarizing, we have obtained that if (51) does not hold, the function $\gamma_{2}=\Psi_{2}\left(\gamma_{1}\right)$ divides the plane into two regions (see Figure 2) such that below and to the right both $|A|_{B P 1}$ and $|A|_{B P 2}$ are positive; above and to the left both are negative.

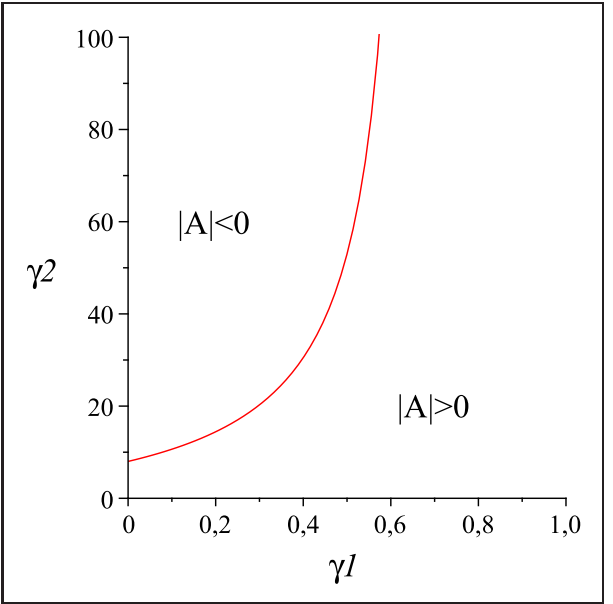

Figure 2: The function $\gamma_{2}=\Psi_{2}\left(\gamma_{1}\right)$ and the corresponding regions in the plane $\left(\gamma_{1}, \gamma_{2}\right)$ where cases (a) or (b) of Lemma 5 hold. Parameter values are $R_{0}^{1}=4, R_{0}^{2}=2, \mu=1$, $\xi_{1}=0.9, \xi_{2}=0.5$.

Since $|A|$ is an affine function of pe (see (46)), if it has the same sign at both ends of a segment, it will have the same sign also within, yielding a) and $b)$. 
To show c), through long computations, one arrives at

$$
\begin{aligned}
& p e_{2}^{B P}-p e_{1}^{B P}=\frac{\left(R_{0}^{1}-R_{0}^{2}\right)\left(R_{0}^{2}\left(1-\xi_{2}\right)-R_{0}^{1}\left(1-\xi_{1}\right)\right) C}{R_{0}^{1} R_{0}^{2}\left(\xi_{1}-\xi_{2}\right)} \\
& \times \frac{\gamma_{2}\left(C\left(\mu+\gamma_{1}\right)-\mu R_{0}^{2} \xi_{2}\right)+\mu R_{0}^{2}\left(\mu R_{0}^{1}\left(\xi_{1}-\xi_{2}\right)+\gamma_{1}\left(R_{0}^{1}\left(\xi_{1}-\xi_{2}\right)+\xi_{2}\right)\right)}{\left(C \gamma_{1}+\mu R_{0}^{1} R_{0}^{2}\left(\xi_{1}-\xi_{2}\right)\right)\left(C \gamma_{2}+\mu R_{0}^{1} R_{0}^{2}\left(\xi_{1}-\xi_{2}\right)\right)} .
\end{aligned}
$$

It is then easy to see that $p e_{2}^{B P}-p e_{1}^{B P}=0$ if and only if $\gamma_{2}=\Psi_{2}\left(\gamma_{1}\right)$.

We can now summarise the conclusions about the existence of the Coexistence Equilibrium.

Proposition 8. Assume $R_{0}^{1}>R_{0}^{2}$ and (36). Then there occur transcritical bifurcations at $E_{1}$ for $p e=p e_{1}^{B P}$, and at $E_{2}$ for $p e=p e_{2}^{B P}$ with the emergence of a positive coexistence equilibrium. Either

(a) $p e_{1}^{B P}<p e_{2}^{B P}$ and the coexistence equilibrium is unique and feasible for all pe $\in\left[p e_{1}^{B P}, p e_{2}^{B P}\right]$;

(b) $p e_{2}^{B P}<p e_{1}^{B P}$ and the coexistence equilibrium is unique and feasible for all pe $\in\left[p e_{2}^{B P}, p e_{1}^{B P}\right]$.

(c) $p e_{2}^{B P}=p e_{1}^{B P}$, and there is a continuum of positive equilibria for $p e=$ $p e_{1}^{B P}=p e_{2}^{B P}$.

If (51) holds, (a) is true for all values of $\gamma_{1}$ and $\gamma_{2}$.

Otherwise, (c) is true for $\gamma_{2}=\Psi_{2}\left(\gamma_{1}\right)$; (b) is true for $\gamma_{2}>\Psi_{2}\left(\gamma_{1}\right)>0$; (a) is true for $\gamma_{2}<\Psi_{2}\left(\gamma_{1}\right)$ and for all $\gamma_{2}$ when $\Psi_{2}\left(\gamma_{1}\right)<0$, where $\Psi_{2}\left(\gamma_{1}\right)$ is defined in (58).

Note that $\Psi_{2}\left(\gamma_{1}\right)>\gamma_{1}$, so that, if $\gamma_{1} \geq \gamma_{2}$, (a) is always true. 


\subsubsection{Stability}

It is easy to show that in case (b) the coexistence equilibrium is always unstable. This can be proved by bifurcation theory, but can also be checked directly using

Lemma 6. Let conditions (36) hold and let $J$ be the Jacobian of (8)-(12) computed at the coexistence equilibrium. Then

$$
\operatorname{sign}(|J|)=\operatorname{sign}(|A|)
$$

The proof is given in the Appendix.

It follows that in case (b), the Routh-Hurwitz stability conditions are violated, and the coexistence equilibrium is unstable.

As for case (a), bifurcation theory shows that the coexistence equilibrium is asymptotically stable for $p e$ close to $p e_{1}^{B P}$ and $p e_{2}^{B P}$.

We were not able to prove that Routh-Hurwitz stability conditions are satisfied for all $p e \in\left(p e_{1}^{B P}, p e_{2}^{B P}\right)$. We then performed a numerical study drawing 1 million sets of parameters $\left(R_{0}^{1}, R_{0}^{2}, \xi_{1}, \xi_{2}, \gamma_{1} / \mu, \gamma_{2} / \mu\right)$ satisfying conditions (36) and (51) or $\Psi_{2}\left(\gamma_{1}\right)<0$ or $\gamma_{2}<\Psi_{2}\left(\gamma_{1}\right)$. For each such draw, we divided the $\left(p e_{1}^{B P}, p e_{2}^{B P}\right)$ interval into 10000 sub-intervals and, for each value of $p e$ in this mesh, computed, through standard routines [16], the eigenvalues of the Jacobian at the coexistence equilibrium. All the computed eigenvalues had negative real parts, suggesting that the coexistence equilibrium never loses its stability through Hopf bifurcations in the intervals $\left(p e_{1}^{B P}, p e_{2}^{B P}\right)$. 


\section{Examples}

The case $p e_{1}^{B P}<p e_{2}^{B P}$ had already been numerically observed by Porco and Blower [1]. In this case coexistence occurs in the parameter region where the other existing equilibria are unstable. The case $p e_{1}^{B P}<p e_{2}^{B P}$ occurs for $\gamma_{1}=\gamma_{2}, \gamma_{1}>\gamma_{2}$ and may occur also for certain $\gamma_{1}<\gamma_{2}$ as shown by the following example.

Example 1: Let $\gamma_{1}=0.015<\gamma_{2}=0.517$ and

$$
R_{0}^{1}=8.363, R_{0}^{2}=3.790, \mu=0.423, \xi_{1}=0.990, \xi_{2}=0.020 \text { and } \pi=1
$$

By substitution into (17), (31) and (41) we get

$$
p e_{1}^{D F}=0.888 \quad p e_{2}^{D F}=36.496 \quad p e_{1}^{B P}=0.153 \quad p e_{2}^{B P}=0.552
$$

The Subtype-1-Only Equilibrium is stable for $0 \leq p e<0.153$, the Subtype2-Only Equilibrium is stable for $0.552<p e \leq 1$ and the Coexistence Equilibrium exists into the range $0.153<p e<0.552$, where the DFE, the Subtype-1-Only and the subtype-2-Only Equilibria exist but are unstable. Numerical computation of the eigenvalues of the linearized system confirm that the Coexistence Equilibrium is stable where it exists. Figure 3 shows the equilibrium fractions $y_{1}$ and $y_{2}$ as function of parameter $p e$. 


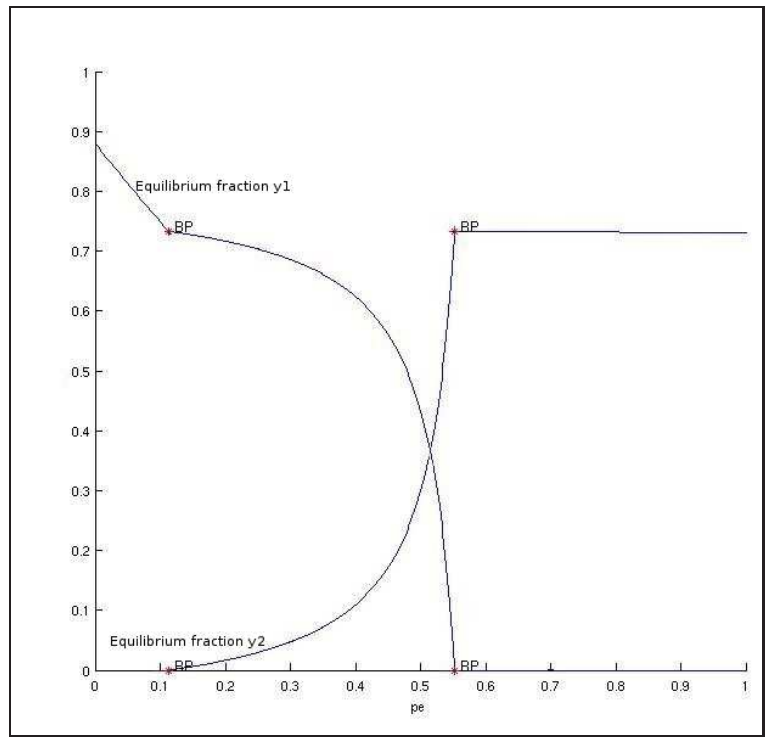

Figure 3: Equilibrium fractions $y_{1}$ and $y_{2}$ as function of pe for fixed $\gamma_{1}=0.015, \gamma_{2}=$ $0.517, R_{0}^{1}=8.363, R_{0}^{2}=3.790, \mu=0.423, \xi_{1}=0.990, \xi_{2}=0.020, \pi=1$. Coexistence of the strains occurs for $0.153<$ pe $<0.552$.

The case $p e_{1}^{B P}>p e_{2}^{B P}$ had never been observed before. In this case the Coexistence Equilibrium exists in a 'bi-stability' region in which both the Subtype-1-Only and the Subtype-2-Only Equilibrium are stable and hence the asymptotic behavior of the system depends on the initial conditions. This latter case occurs only for certain $\gamma_{1}<\gamma_{2}$.

Example 2: Consider the case $\gamma_{1}=0.026<\gamma_{2}=0.966$ and let

$$
R_{0}^{1}=4.723, R_{0}^{2}=2.293, \mu=0.235, \xi_{1}=0.923, \xi_{2}=0.650 \text { and } \pi=1
$$

By substitution into (17), (31) and (41) we get

$$
p e_{1}^{D F}=0.853 \quad p e_{2}^{D F}=0.866 \quad p e_{1}^{B P}=0.829 \quad p e_{2}^{B P}=0.822
$$

The DFE is stable for $p e>0.866$, the Subtype-1-Only Equilibrium is stable for $0<p e<0.829$ and the Subtype-2-Only Equilibrium is stable for $0.822<$ 
$p e<0.866$. The Coexistence Equilibrium exists for $0.822<p e<0.829$ and is unstable. Figure 4 shows the equilibrium fractions $y_{1}$ and $y_{2}$ as function of parameter pe. Figure 5 shows two trajectories for the equilibrium fractions $y_{1}$ and $y_{2}$ starting close to the Coexistence Equilibrium at $p e=0.8234$ and converging one to the Subtype-1-Only Equilibrium and the other to the Subtype-2-Only Equilibrium. The bifurcation and trajectory graphs have been obtained by the graphical package MatCont of the MATLAB software.

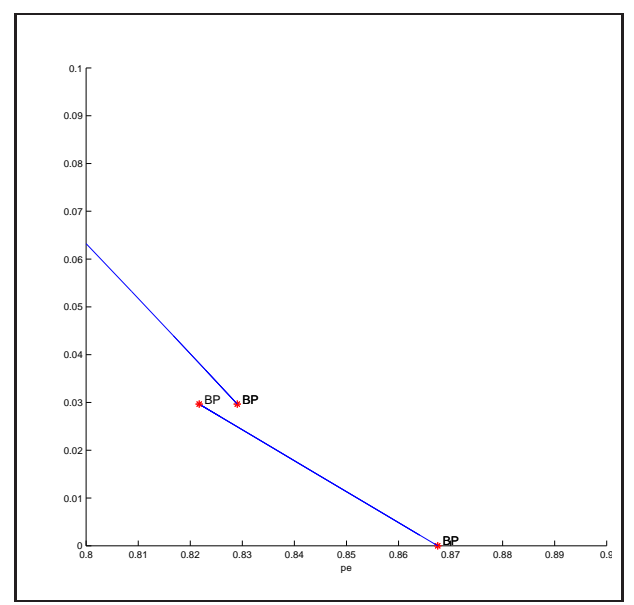

Figure 4: Equilibrium fractions $y_{1}$ and $y_{2}$ as function of pe for fixed $\gamma_{1}=0.026, \gamma_{2}=$ $0.966, R_{0}^{1}=4.723, R_{0}^{2}=2.293, \mu=0.235, \xi_{1}=0.923, \xi_{2}=0.650, \pi=1$. A bi-stability region occurs for $0.822<p e<0.829$; in this region unstable coexistence of the strains occurs. 

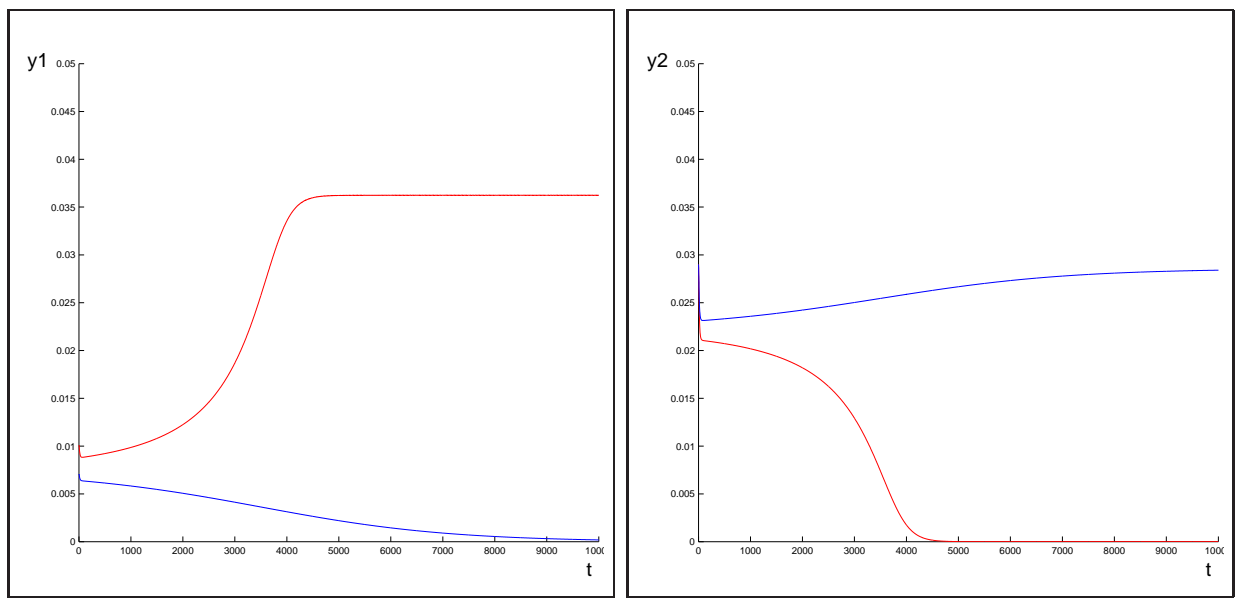

Figure 5: Trajectories of the fractions $y_{1}$ (left panel) and $y_{2}$ (right panel) as functions of time; parameter values are $\gamma_{1}=0.026, \gamma_{2}=0.966, R_{0}^{1}=4.723, R_{0}^{2}=2.293, \mu=0.235, \xi_{1}=$ $0.923, \xi_{2}=0.650, \pi=1$ and $p e=0.8234$. Both trajectories start close to the Coexistence Equilibrium $x=0.148, y_{1}=0.007, y_{2}=0.022, N=3.896$; the starting point of the red one, converging to the Subtype-1-Only Equilibrium, is $(0.148,0.010,0.029,3.896)$; the starting point of the blue one, converging to the Subtype-2-Only Equilibrium, is $(0.148,0.007,0.029,3.896)$.

\section{Discussion}

In this manuscript we have analysed a model for competition between two viral strains with complete cross-immunity and imperfect vaccination. The model was first proposed by Porco and Blower [1] for different HIV strains; the authors showed through simulations the possibility that vaccination shifted the competitive hierarchy, with potential side-effects on public health.

Here we have examined the same model in greater detail, finding for instance the exact conditions under which vaccination may lead to coexistence of two strains; these are given by (36). It is worth commenting on their biological interpretation.

The first $\xi_{1}>\xi_{2}$ means that the vaccine reduces more the susceptibility to the strain with the higher reproduction number (the better competitor in absence of vaccination) since we assumed $R_{0}^{1}>R_{0}^{2}$. 
The second condition $p e_{2}^{D F}>p e_{1}^{D F}$ specifies that it is harder to eradicate through vaccination strain 2 than strain 1 , if they are the only strains present. Writing the condition as

$$
\frac{\xi_{2}}{\xi_{1}}<\frac{R_{0}^{1}\left(R_{0}^{2}-1\right)}{R_{0}^{2}\left(R_{0}^{1}-1\right)}
$$

it specifies that the ratio of susceptibilities under vaccination must be decreased sufficiently relative to a ratio of reproduction numbers.

The third condition $R_{0}^{2}\left(1-\xi_{2}\right)>R_{0}^{1}\left(1-\xi_{1}\right)$ means that, if every individual were vaccinated, the second strain would have a higher reproduction number (note that the third condition implies the first one, which is then pleonastic).

Under these conditions there always exists a range of vaccination rates under which a (unique) coexistence equilibrium exists, at least if $\mu / \gamma_{i}$ is small enough, i.e. natural mortality is sufficiently lower than that induced by the infection (or, in case of HIV, than the rate of progressing into AIDS).

The relative values of $\gamma_{1}$ and $\gamma_{2}$ (i.e., of the expected lengths of sojourn in the classes $I_{1}$ and $I_{2}$ ) determine the ordering between $p e_{1}^{B P}$ given by (31) and $p e_{2}^{B P}$ given by (41). This in turn affects the qualitative behavior of system (14).

The case $p e_{1}^{B P}<p e_{2}^{B P}$ had already been numerically observed [1]. In this case, coexistence occurs in the parameter region where all the other equilibria are unstable. Numerically, we found that the coexistence equilibrium is asymptotically stable for parameter values in this region, but the possibility of destabilization via Hopf bifurcations cannot be totally excluded, since an analytical proof is missing. The unconditional stability of the coexistence equilibrium has been proven in another model with coexistence of totally 
cross-immune pathogen strains [17].

On the other hand, the case $p e_{1}^{B P}>p e_{2}^{B P}$ is also possible, giving rise to phenomena that had not been anticipated. In this case there exists a parameter region in which both monomorphic equilibria (i.e. the Subtype1-Only and the Subtype-2-Only Equilibrium) are stable and the coexistence equilibrium exists unstable (see Figure 4). In this 'bi-stability' region the asymptotic behavior of system (8)-(12) depends on the initial conditions. The presence of the bi-stability region implies that, with a gradual increase of vaccination rates, one may encounter a sudden shift from a situation with only strain 1 present in appreciable proportion, to one with only strain 2 . Moreover, decreasing again vaccination rates, one would see a hysteresistype behavior.

As shown in the main text, bi-stability may occur only if $\gamma_{1}<\gamma_{2}$. This means that the mortality rate (or rate of developing AIDS, in case of HIV) must be larger for strain 2 (the one that is out-competed without vaccination) than for strain 1 . In other words, bi-stability may occur only if vaccination shifts the competitive balance in favour of a more virulent strain, a rather unpleasant scenario [18].

The results obtained in this work depend on the simplifying assumption that co-infection does not occur (i.e. the vaccine confers complete crossprotection). Several models have been proposed that weaken this assumption; the simplest alternative is to allow for super-infection $[6,22]$, i.e. an individual infected with one strain gets infected with another strain which may lead to an instantaneous (if we neglect the inevitable phase during which the two strains will coexist in the individual) replacement of the intial strain. It has been known for a long time [21] that with super-infection strain coexistence is possible even without vaccination; in this case, strain 
replacement may be induced by vaccination even without the vaccine being differential effective [7]. This case bears some differences from the one examined here, but it can be expected that strain coexistence or exclusion can be analysed along the same lines.

Much more intricate is the case with co-infection, i.e. an individual is infected with several strains at the same time, whether this occurs because of new infections [22] or because of within-host virus mutations, as usual for HIV [23]. It is intuitive that, in this case, strain coexistence without vaccination would be usual, but mathematical models accounting for such phenomena, and amenable to analytical methods, are still at a preliminary stage [24]. Exploring the effect of a vaccine with differential effectiveness in a model allowing for co-infection is a fundamental and fascinating issue, but goes beyond the scope of this work and the techniques used here.

The model is definitely not realistic for HIV, mainly because its structure implies that the duration of the infectious stage is exponential, which is certainly not plausible, whether infectious are treated or not. However, the results obtained in this paper can be generalized, at least as far as the existence of equilibria is concerned, to more realistic distributions for the infectious period, as we show next.

Let $\gamma_{i}(\theta)$ be the exit rate from the class $Y_{i}$ for individuals that have spent time $\theta$ in the class. This corresponds to a distribution of infectious period $T_{i}$ given by

$$
P\left(T_{i} \geq \theta\right)=\Gamma_{i}(\theta):=\exp \left\{-\int_{0}^{\theta} \gamma_{i}(s) d s\right\}
$$

conditional on having survived natural mortality. Assume also that the infectiousness depends on the time since infection through a function $\phi(\theta)$.

Denote $Y_{i}(t, \theta)$ the number of individuals infected at time $t$ by Subtype-i 
for a time period of length $\theta$ and let's set

$$
Q_{i}(t)=\int_{0}^{+\infty} \phi(\theta) Y_{i}(t, \theta) d \theta
$$

Analogously to (1)-(4), we assume that the rate of new infections with Subtype-i (with $i=1,2$ ) at time $t$ is given by

$$
c \beta_{i} X(t) \frac{Q_{i}(t)}{N(t)}
$$

Under these assumptions, the dynamics described in Figure 1 lead to

$$
\begin{aligned}
\dot{X} & =\pi(1-p e)-\mu X-c \beta_{1} X \frac{Q_{1}}{N}-c \beta_{2} X \frac{Q_{2}}{N} \\
\dot{V} & =\pi p e-\mu V-\left(1-\xi_{1}\right) c \beta_{1} V \frac{Q_{1}}{N}-\left(1-\xi_{2}\right) c \beta_{2} V \frac{Q_{2}}{N} \\
\frac{\partial Y_{1}}{\partial t}+\frac{\partial Y_{1}}{\partial \theta} & =-\left(\mu+\gamma_{1}(\theta)\right) Y_{1}(t, \theta) \\
\frac{\partial Y_{2}}{\partial t}+\frac{\partial Y_{2}}{\partial \theta} & =-\left(\mu+\gamma_{2}(\theta)\right) Y_{2}(t, \theta) \\
\dot{N} & =\pi-\mu N-\int_{0}^{+\infty} \gamma_{1}(\theta) Y_{1}(t, \theta) d \theta-\int_{0}^{+\infty} \gamma_{2}(\theta) Y_{2}(t, \theta) d \theta
\end{aligned}
$$

where

$$
N=X+V+\int_{0}^{+\infty} Y_{1}(t, \theta) d \theta+\int_{0}^{+\infty} Y_{2}(t, \theta) d \theta
$$

and with

$$
Y_{i}(t, 0)=c \beta_{i} \frac{Q_{i}}{N}\left(X+\left(1-\xi_{i}\right) V\right), \quad i=1,2 .
$$

We show here that, under a proper re-parameterization, an equilibrium solution of (61)-(67) satisfies equations (15), (43) and (45).

Let's denote the equilibrium fractions of susceptible, vaccinated and infected individuals respectively by

$$
\tilde{x}=\frac{\tilde{X}}{\tilde{N}}, \quad \tilde{v}=\frac{\tilde{V}}{\tilde{N}} \quad \text { and } \quad \tilde{y}_{i}=\frac{\tilde{\Lambda}_{i}}{\tilde{N}}
$$


where

$$
\tilde{\Lambda}_{i}=c \beta_{i} \frac{\tilde{Q}_{i}}{\tilde{N}}\left(\tilde{X}+\left(1-\xi_{i}\right) \tilde{V}\right), \quad i=1,2 .
$$

A solution of (63)-(64) independent of $t$ is given by

$$
\tilde{Y}_{i}(\theta)=\tilde{\Lambda}_{i} e^{-\mu \theta} \Gamma_{i}(\theta), \quad i=1,2
$$

where $\Gamma_{i}(\theta)$ is defined in (59). By substitution of (70) into (65), we obtain that a solution of (61)-(65) has to satisfy equation (15), using $\tilde{\gamma}_{i}=$ $\int_{0}^{+\infty} \gamma_{i}(\theta) e^{-\mu \theta} \Gamma_{i}(\theta) d \theta$ in place of $\gamma_{i}, i=1,2$.

From (68) and (60) it follows that

$$
\frac{\tilde{Q}_{i}}{\tilde{N}}=\frac{\tilde{\Lambda}_{i}}{\tilde{N}} \int_{0}^{+\infty} \phi(\theta) e^{-\mu \theta} \Gamma_{i}(\theta) d \theta
$$

From (69) it follows that

$$
\tilde{\Lambda}_{i}=c \beta_{i} \frac{\tilde{Q}_{i}}{\tilde{N}}\left(\tilde{X}+\left(1-\xi_{i}\right) \tilde{V}\right), \quad i=1,2
$$

and by substitution of (71) into (72) one obtains that a steady state has to satisfy equations (43), where $R_{0}^{i}=c \beta_{i} \int_{0}^{+\infty} \phi(\theta) e^{-\mu \theta} \Gamma_{i}(\theta) d \theta, i=1,2$. Finally, one can show that equations (61)-(62) can be written as (45).

Thus, using the parameterization and the new definitions given here, all results concerning existence of equilibria are still valid. Stability is more delicate: Thieme and Castilo-Chavez [19] have proved that, with variable infectiousness, the endemic equilibrium can be unstable even with a single strain and no vaccination.

The model can also be applied to many other diseases of S-I type caused by infectious agents circulating (at a population-level) in multiple-types or sub-types, as long as one can assume that the entrance in the community 
(with or without vaccination) is constant. It is a reasonable assumption at least for time-scales of the order of several years. For instance, system (14) could be well suited to study the spread of two of the nearly 200 identified types of Human Papilloma Virus (HPV) at a population-level [20]. Of course the model should be adapted to the specific disease taken into consideration; in the case of HPV, for instance, we should account for the fact that mortality due to infection is negligible and that immunity may be lost, thus slightly changing the structure of the model. The exact results would then depend on the appropriate model chosen, but we expect our analysis to be of help in shedding light on the behaviour of similar models and to be of interest for the analysis of several emerging and re-emerging fatal infectious diseases.

\section{Acknowledgments}

The authors thank prof. Sally Blower for her suggestions. Her kind supervision to ID's Master's Thesis has been the first step that led to this work. The authors also thank two anonymous referees for several useful comments and suggestions that helped improve the presentation. 


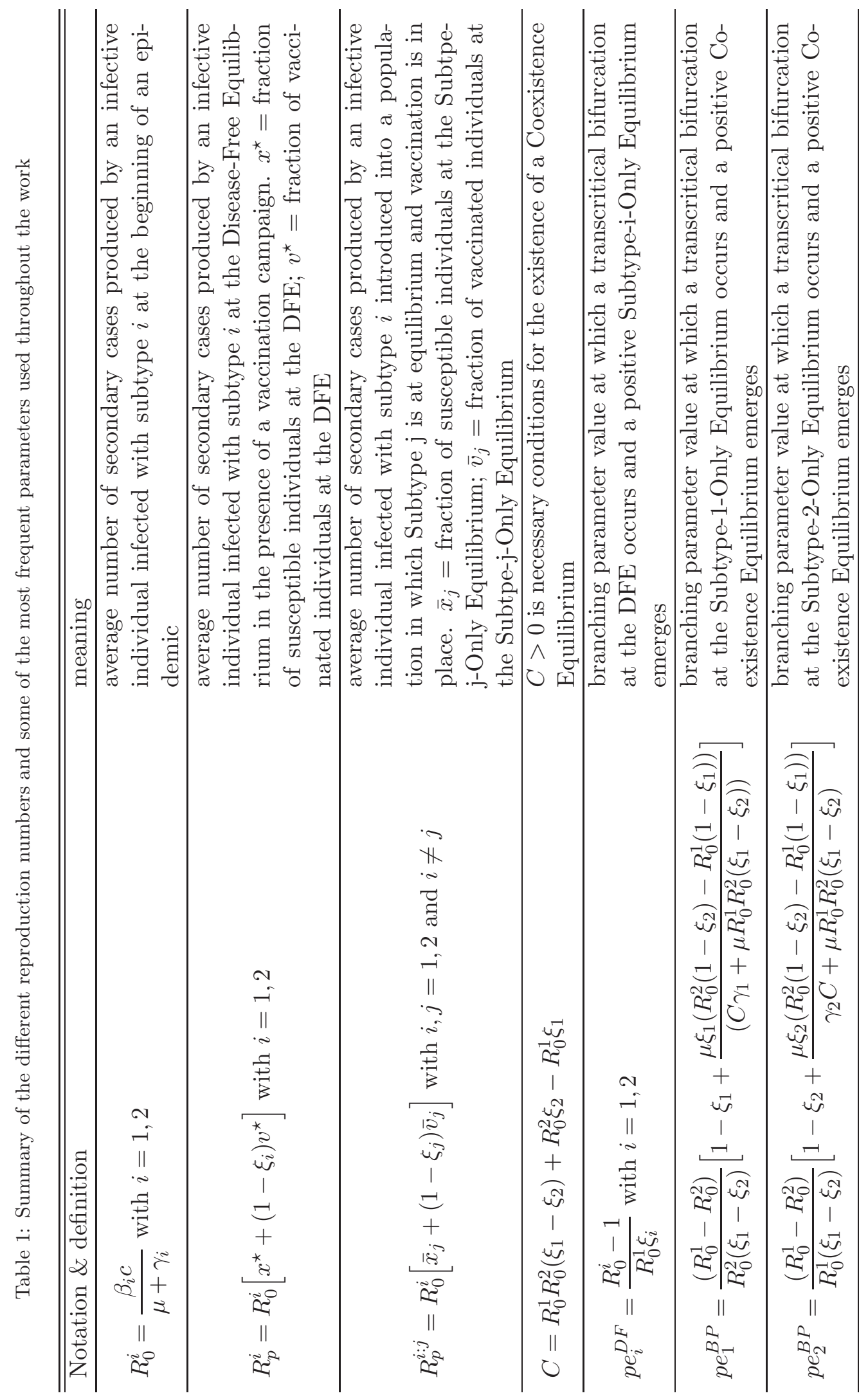




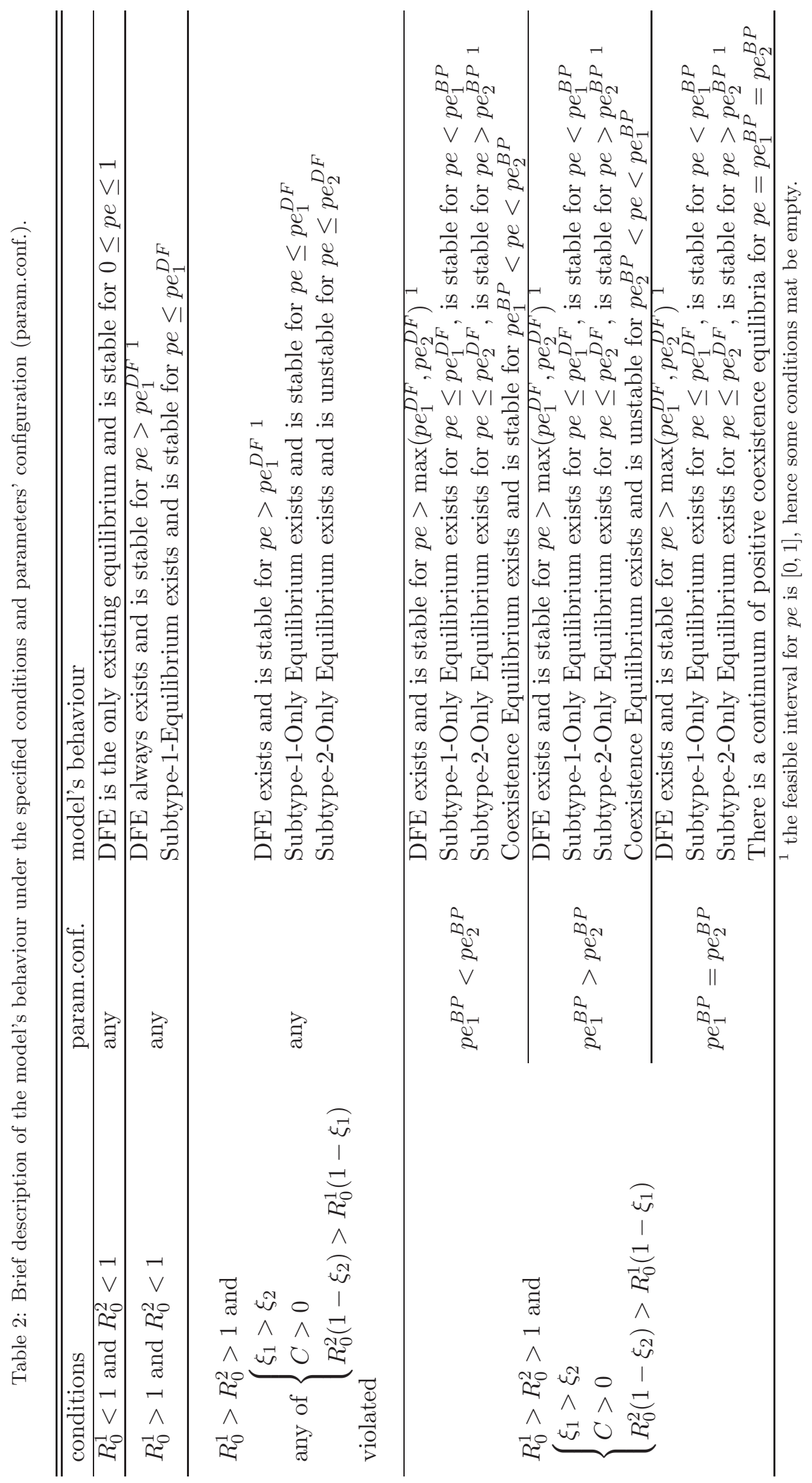




\section{A Appendix}

\section{A.1 Proof of Lemma 1}

Proof. The characteristic polynomial of $E$ (after a change of sign) can be written as

$$
\lambda^{3}+a_{1} \lambda^{2}+a_{2} \lambda+a_{3}=0
$$

Routh-Hurwitz criterion states that all solutions of (73) have negative real part if and only if $a_{1}, a_{2}, a_{3}>0$ and $a_{1} a_{2}-a_{3}>0$ [14].

If we set

$$
\begin{aligned}
K & =R_{0}^{1}\left(\mu+\gamma_{1}\right)\left(1-\xi_{1}\right) \bar{y}_{1} \\
L & =R_{0}^{1}\left(\mu+\gamma_{1}\right) \bar{y}_{1}
\end{aligned}
$$

after some computations (see section "Computation of the Routh-Hurwitz coefficients" below) we obtain

$$
\begin{aligned}
a_{1}= & 2 \mu+K+L \\
a_{2}= & (\mu+L)(\mu+K)+\left[R_{0}^{1}\left(\mu+\gamma_{1}\right)-\gamma_{1}\right] L \xi_{1} \bar{x}_{1}+\left(\mu+\gamma_{1} \bar{y}_{1}\right) K \\
a_{3}= & {\left[R_{0}^{1}\left(\mu+\gamma_{1}\right)-\gamma_{1}\right] L \mu \xi_{1} \bar{x}_{1}+\left(\mu+\gamma_{1} \bar{y}_{1}\right)(\mu+L) K } \\
a_{1} a_{2}-a_{3}= & (\mu+L)(\mu+K)(2 \mu+L+K)+\left[R_{0}^{1}\left(\mu+\gamma_{1}\right)-\gamma_{1}\right] L \xi_{1} \bar{x}_{1}(\mu+L+K)+ \\
& +\left(\mu+\gamma_{1} \bar{y}_{1}\right)(\mu+K) K .
\end{aligned}
$$

Since $R_{0}^{1}>1$ and hence $R_{0}^{1}\left(\mu+\gamma_{1}\right)>\gamma_{1}$ all these quantities are positive, thus proving that Routh-Hurwitz conditions are satisfied. 


\section{A.1.1 Computation of the Routh-Hurwitz coefficients}

We report here the computations of $a_{1}, a_{2}, a_{3}$ and $a_{1} a_{2}-a_{3}$ that lead us to prove that the Subtype-1-Only Equilibrium is stable, wherever it exists.

Remind that we set

$$
\begin{aligned}
K & =R_{0}^{1}\left(\mu+\gamma_{1}\right)\left(1-\xi_{1}\right) \bar{y}_{1} \\
L & =R_{0}^{1}\left(\mu+\gamma_{1}\right) \bar{y}_{1}
\end{aligned}
$$

The following equalities hold

$$
\begin{aligned}
a_{1} & =\left(\mu+\gamma_{1} \bar{y}_{1}\right)+\left[\mu+R_{0}^{1}\left(\mu+\gamma_{1}\right) \bar{y}_{1}\right]-\gamma_{1} \bar{y}_{1}+R_{0}^{1}\left(\mu+\gamma_{1}\right)\left(1-\xi_{1}\right) \bar{y}_{1} \\
& =\mu+\mu+R_{0}^{1}\left(\mu+\gamma_{1}\right) \bar{y}_{1}+R_{0}^{1}\left(\mu+\gamma_{1}\right)\left(1-\xi_{1}\right) \bar{y}_{1} \\
& =2 \mu+K+L
\end{aligned}
$$

$$
\begin{aligned}
a_{2} & =\left(\mu+\gamma_{1} \bar{y}_{1}\right)\left[\mu+R_{0}^{1}\left(\mu+\gamma_{1}\right) \bar{y}_{1}\right]-\left[\mu+R_{0}^{1}\left(\mu+\gamma_{1}\right) \bar{y}_{1}\right]\left[\gamma_{1} \bar{y}_{1}-R_{0}^{1}\left(\mu+\gamma_{1}\right)\left(1-\xi_{1}\right) \bar{y}_{1}\right] \\
& +\left[R_{0}^{1}\left(\mu+\gamma_{1}\right)-\gamma_{1}\right] R_{0}^{1}\left(\mu+\gamma_{1}\right) \xi_{1} \bar{x}_{1} \bar{y}_{1}-\left(\mu+\gamma_{1} \bar{y}_{1}\right)\left[\gamma_{1} \bar{y}_{1}-R_{0}^{1}\left(\mu+\gamma_{1}\right)\left(1-\xi_{1}\right) \bar{y}_{1}\right]+\left(\mu+\gamma_{1} \bar{y}_{1}\right) \gamma_{1} \bar{y}_{1} \\
& =\left[\mu+R_{0}^{1}\left(\mu+\gamma_{1}\right) \bar{y}_{1}\right]\left[\mu+R_{0}^{1}\left(\mu+\gamma_{1}\right)\left(1-\xi_{1}\right) \bar{y}_{1}\right]+\left[R_{0}^{1}\left(\mu+\gamma_{1}\right)-\gamma_{1}\right] R_{0}^{1}\left(\mu+\gamma_{1}\right) \xi_{1} \bar{x}_{1} \bar{y}_{1}+ \\
& +\left(\mu+\gamma_{1} \bar{y}_{1}\right) R_{0}^{1}\left(\mu+\gamma_{1}\right)\left(1-\xi_{1}\right) \bar{y}_{1} \\
& =(\mu+L)(\mu+K)+\left[R_{0}^{1}\left(\mu+\gamma_{1}\right)-\gamma_{1}\right] L \xi_{1} \bar{x}_{1}+\left(\mu+\gamma_{1} \bar{y}_{1}\right) K
\end{aligned}
$$

$$
\begin{aligned}
a_{3} & =\left(\mu+\gamma_{1} \bar{y}_{1}\right)\left\{\left[R_{0}^{1}\left(\mu+\gamma_{1}\right)-\gamma_{1}\right] R_{0}^{1}\left(\mu+\gamma_{1}\right) \xi_{1} \bar{x}_{1} \bar{y}_{1}-\left[\mu+R_{0}^{1}\left(\mu+\gamma_{1}\right) \bar{y}_{1}\right]\left[\gamma_{1} \bar{y}_{1}+\right.\right. \\
& \left.\left.-R_{0}^{1}\left(\mu+\gamma_{1}\right)\left(1-\xi_{1}\right) \bar{y}_{1}\right]\right\}+\gamma_{1} \bar{N}\left[\gamma_{1} \bar{y}_{1}-R_{0}^{1}\left(\mu+\gamma_{1}\right) \bar{y}_{1}\right] R_{0}^{1}\left(\mu+\gamma_{1}\right) \xi_{1} \frac{\bar{x}_{1} \bar{y}_{1}}{\bar{N}}+ \\
& \left.+\left(\mu+\gamma_{1} \bar{y}_{1}\right)\left[\mu+R_{0}^{1}\left(\mu+\gamma_{1}\right) \bar{y}_{1}\right] \frac{\bar{y}_{1}}{\bar{N}}\right] \\
& =\left(\mu+\gamma_{1} \bar{y}_{1}\right)\left\{\left[R_{0}^{1}\left(\mu+\gamma_{1}\right)-\gamma_{1}\right] R_{0}^{1}\left(\mu+\gamma_{1}\right) \xi_{1} \bar{x}_{1} \bar{y}_{1}-\left[\mu+R_{0}^{1}\left(\mu+\gamma_{1}\right) \bar{y}_{1}\right]\left[\gamma_{1} \bar{y}_{1}+\right.\right. \\
& \left.\left.-R_{0}^{1}\left(\mu+\gamma_{1}\right)\left(1-\xi_{1}\right) \bar{y}_{1}\right]\right\}-\left[R_{0}^{1}\left(\mu+\gamma_{1}\right)-\gamma_{1}\right] R_{0}^{1}\left(\mu+\gamma_{1}\right) \xi_{1} \bar{x}_{1} \bar{y}_{1} \gamma_{1} \bar{y}_{1}+ \\
& +\left(\mu+\gamma_{1} \bar{y}_{1}\right)\left[\mu+R_{0}^{1}\left(\mu+\gamma_{1}\right) \bar{y}_{1}\right] \gamma_{1} \bar{y}_{1} \\
& =\left[R_{0}^{1}\left(\mu+\gamma_{1}\right)-\gamma_{1}\right] L \mu \xi_{1} \bar{x}_{1}+\left(\mu+\gamma_{1} \bar{y}_{1}\right)(\mu+L) K
\end{aligned}
$$




$$
\begin{aligned}
a_{1} a_{2}-a_{3} & =\left[\mu+R_{0}^{1}\left(\mu+\gamma_{1}\right) \bar{y}_{1}\right]\left[\mu+R_{0}^{1}\left(\mu+\gamma_{1}\right)\left(1-\xi_{1}\right) \bar{y}_{1}\right]\left[2 \mu+R_{0}^{1}\left(\mu+\gamma_{1}\right) \bar{y}_{1}+\right. \\
+ & \left.R_{0}^{1}\left(\mu+\gamma_{1}\right)\left(1-\xi_{1}\right) \bar{y}_{1}\right]+\left[R_{0}^{1}\left(\mu+\gamma_{1}\right)-\gamma_{1}\right] R_{0}^{1}\left(\mu+\gamma_{1}\right) \xi_{1} \bar{x}_{1} \bar{y}_{1}[2 \mu+ \\
& \left.+R_{0}^{1}\left(\mu+\gamma_{1}\right) \bar{y}_{1}+R_{0}^{1}\left(\mu+\gamma_{1}\right)\left(1-\xi_{1}\right) \bar{y}_{1}\right]+\left(\mu+\gamma_{1} \bar{y}_{1}\right) R_{0}^{1}\left(\mu+\gamma_{1}\right)\left(1-\xi_{1}\right) \bar{y}_{1}[2 \mu+ \\
& \left.R_{0}^{1}\left(\mu+\gamma_{1}\right) \bar{y}_{1}+R_{0}^{1}\left(\mu+\gamma_{1}\right)\left(1-\xi_{1}\right) \bar{y}_{1}\right]-\left[R_{0}^{1}\left(\mu+\gamma_{1}\right)-\gamma_{1}\right] R_{0}^{1}\left(\mu+\gamma_{1}\right) \xi_{1} \bar{x}_{1} \bar{y}_{1}+ \\
& -\left(\mu+\gamma_{1} \bar{y}_{1}\right)\left[\mu+R_{0}^{1}\left(\mu+\gamma_{1}\right) \bar{y}_{1}\right] R_{0}^{1}\left(\mu+\gamma_{1}\right)\left(1-\xi_{1}\right) \bar{y}_{1} \\
& =(\mu+L)(\mu+K)(2 \mu+L+K)+\left[R_{0}^{1}\left(\mu+\gamma_{1}\right)-\gamma_{1}\right] L \xi_{1} \bar{x}_{1}(\mu+L+K)+ \\
& +\left(\mu+\gamma_{1} \bar{y}_{1}\right)(\mu+K) K
\end{aligned}
$$

\section{A.2 Proof of Lemma 2}

Proof. a) It is clear that, under the assumptions (29), if $R_{0}^{2}\left(1-\xi_{2}\right)>R_{0}^{1}\left(1-\xi_{1}\right)$, then $0<p e_{1}^{B P}$. As for the other inequality, if $\mu=0$,

$$
p e_{1}^{B P}=\frac{\left(R_{0}^{1}-R_{0}^{2}\right)\left(1-\xi_{1}\right)}{R_{0}^{2}\left(\xi_{1}-\xi_{2}\right)}
$$

and

$$
\frac{\left(R_{0}^{1}-R_{0}^{2}\right)\left(1-\xi_{1}\right)}{R_{0}^{2}\left(\xi_{1}-\xi_{2}\right)}<1 \Longleftrightarrow R_{0}^{1}\left(1-\xi_{1}\right)<R_{0}^{2}\left(1-\xi_{2}\right) .
$$

By continuity, if $R_{0}^{2}\left(1-\xi_{2}\right)>R_{0}^{1}\left(1-\xi_{1}\right), p e_{1}^{B P}<1$ for $\mu>0$ small enough.

b) If $R_{0}^{2}\left(1-\xi_{2}\right)=R_{0}^{1}\left(1-\xi_{1}\right), p e_{1}^{B P} \equiv 1$ for all $\mu>0$.

If $R_{0}^{2}\left(1-\xi_{2}\right)<R_{0}^{1}\left(1-\xi_{1}\right), p e_{1}^{B P}$ is a decreasing continuous function of $\mu$ on $[0,+\infty)$.

Hence

$$
p e_{1}^{B P}>p_{\infty}:=\lim _{\mu \rightarrow+\infty} p e_{1}^{B P}=\frac{\left(R_{0}^{1}-R_{0}^{2}\right)}{R_{0}^{2}\left(\xi_{1}-\xi_{2}\right)}\left[1-\xi_{1}+\frac{\xi_{1}\left(R_{0}^{2}\left(1-\xi_{2}\right)-R_{0}^{1}\left(1-\xi_{1}\right)\right)}{R_{0}^{1} R_{0}^{2}\left(\xi_{1}-\xi_{2}\right)}\right] .
$$

Now

$$
\begin{gathered}
p_{\infty}-1=\frac{\left(R_{0}^{1}-R_{0}^{2}\right)\left(1-\xi_{1}\right)-R_{0}^{2}\left(\xi_{1}-\xi_{2}\right)}{R_{0}^{2}\left(\xi_{1}-\xi_{2}\right)}+\frac{\left(R_{0}^{1}-R_{0}^{2}\right) \xi_{1}\left(R_{0}^{2}\left(1-\xi_{2}\right)-R_{0}^{1}\left(1-\xi_{1}\right)\right)}{R_{0}^{2}\left(\xi_{1}-\xi_{2}\right) R_{0}^{1} R_{0}^{2}\left(\xi_{1}-\xi_{2}\right)} \\
=\frac{R_{0}^{1}\left(1-\xi_{1}\right)-R_{0}^{2}\left(1-\xi_{2}\right)}{R_{0}^{2}\left(\xi_{1}-\xi_{2}\right)}\left(1-\frac{\left(R_{0}^{1}-R_{0}^{2}\right) \xi_{1}}{R_{0}^{1} R_{0}^{2}\left(\xi_{1}-\xi_{2}\right)}\right) .
\end{gathered}
$$


We use the inequality $\left(R_{0}^{1}-R_{0}^{2}\right) \xi_{1}<R_{0}^{1} \xi_{1}-R_{0}^{2} \xi_{2}$ in the bracketed term to have

$$
1-\frac{\left(R_{0}^{1}-R_{0}^{2}\right) \xi_{1}}{R_{0}^{1} R_{0}^{2}\left(\xi_{1}-\xi_{2}\right)}>1-\frac{R_{0}^{1} \xi_{1}-R_{0}^{2} \xi_{2}}{R_{0}^{1} R_{0}^{2}\left(\xi_{1}-\xi_{2}\right)}=\frac{C}{R_{0}^{1} R_{0}^{2}\left(\xi_{1}-\xi_{2}\right)}>0
$$

proving $p_{\infty}-1>0$.

\section{A.3 Proof of Lemma 6}

Proof. Let $J$ be the Jacobian matrix at the coexistence equilibrium:

$J=\left(\begin{array}{cccc}-\frac{\pi}{\hat{N}} & 0 & -\gamma_{1} \hat{N} & -\gamma_{2} \hat{N} \\ -\frac{\pi}{\hat{N}^{2}}(1-\hat{x}-p e) & -\frac{\pi}{\hat{N}}-a & -\hat{x}\left[R_{0}^{1}\left(\mu+\gamma_{1}\right)-\gamma_{1}\right] & -\hat{x}\left[R_{0}^{2}\left(\mu+\gamma_{2}\right)-\gamma_{2}\right] \\ \frac{\pi}{\hat{N}^{2}} \hat{y}_{1} & \hat{y}_{1} R_{0}^{1}\left(\mu+\gamma_{1}\right) \xi_{1} & -\hat{y}_{1} b+\gamma_{1} \hat{y}_{1} & -\hat{y}_{1} b+\gamma_{2} \hat{y}_{1} \\ \frac{\pi}{\hat{N}^{2}} \hat{y}_{2} & \hat{y}_{2} R_{0}^{2}\left(\mu+\gamma_{2}\right) \xi_{2} & -\hat{y}_{2} c+\gamma_{1} \hat{y}_{2} & -\hat{y}_{2} c+\gamma_{2} \hat{y}_{2}\end{array}\right)$

where

$$
\begin{aligned}
& a=R_{0}^{1}\left(\mu+\gamma_{1}\right) \hat{y}_{1}-\gamma_{1} \hat{y}_{1}+R_{0}^{2}\left(\mu+\gamma_{2}\right) \hat{y}_{2}-\gamma_{2} \hat{y}_{2} \\
& b=R_{0}^{1}\left(\mu+\gamma_{1}\right)\left(1-\xi_{1}\right) \\
& c=R_{0}^{2}\left(\mu+\gamma_{2}\right)\left(1-\xi_{2}\right)
\end{aligned}
$$

We apply the Gauss-Jordan algorithm in the following steps:

1) substitute the fourth row of $J$ with the sum of its fourth row multiplied times $\hat{y}_{1}$ and its third row multiplied times $-\hat{y}_{2}$, thus obtaining matrix $J_{1}$

2) substitute the third column of matrix $J_{1}$ with the sum of the its thrid column and its fourth column multiplied times -1 , thus obtaining matrix $J_{2}$

3) substitute the third row of matrix $J_{2}$ with the sum of its third row multiplied times $\hat{N}$ and its first row multiplied times $\hat{y}_{1}$, thus obtaining matrix

$$
J_{3}=\left(\begin{array}{cccc}
-\frac{\pi}{\hat{N}} & 0 & \left(\gamma_{2}-\gamma_{1}\right) \hat{N} & -\gamma_{2} \hat{N} \\
-\frac{\pi}{\hat{N}^{2}}(1-\hat{x}-p e) & -\frac{\pi}{\hat{N}}-a & \hat{x} d & -\hat{x}\left[R_{0}^{2}\left(\mu+\gamma_{2}\right)-\gamma_{2}\right] \\
0 & \hat{N} R_{0}^{1} \hat{y}_{1}\left(\mu+\gamma_{1}\right) \xi_{1} & 0 & -\hat{N} R_{0}^{1} \hat{y}_{1}\left(\mu+\gamma_{1}\right)\left(1-\xi_{1}\right) \\
0 & \hat{y}_{1} \hat{y}_{2} e & 0 & \hat{y}_{1} \hat{y}_{2}(b-c)
\end{array}\right) .
$$


where $a, b$ and $c$ are given in (74) and

$$
\begin{aligned}
& d=R_{0}^{2}\left(\mu+\gamma_{2}\right)-\gamma_{2}-R_{0}^{1}\left(\mu+\gamma_{1}\right)+\gamma_{1} \\
& e=R_{0}^{2}\left(\mu+\gamma_{2}\right) \xi_{2}-R_{0}^{1}\left(\mu+\gamma_{1}\right) \xi_{1}
\end{aligned}
$$

Due to the properties of the determinant, we have

$$
|J|=\left|J_{3}\right| .
$$

We compute $\left|J_{3}\right|$ expanding through its first column obtaining

$$
\left|J_{3}\right|=\frac{|A| \pi R_{0}^{1} R_{0}^{2}\left(\mu+\gamma_{1}\right)\left(\mu+\gamma_{2}\right) \hat{y}_{1}^{2} \hat{y}_{2}\left(\xi_{1}-\xi_{2}\right)}{\mu}
$$

where $|A|$ is given in (46). Conditions (36) and identities (75) and (76) imply our claim. 


\section{References}

[1] T. C. Porco and S. Blower. Designing HIV vaccination policies: Subtypes and cross-immunity. Interfaces, 23:167-190, 1998.

[2] H.J. Bremermann and H.R. Thieme. A competitive exclusion principle for pathogen virulence. J. Math. Biol., 27:179-190, 1989.

[3] A.R. McLean. Vaccination, evolution and changes in the efficacy of vaccine - a theoretical framework. Proceedings of the Royal Society of London Series B-Biological Sciences, 261:389-393, 1995.

[4] M. Martcheva, B.M. Bolker, and R.D. Holt. Vaccine-induced pathogen strain replacement: what are the mechanisms? Journal of the Royal Society, 5- No. 18:3-13, 2008.

[5] M. Lipsitch. Bacterial vaccines and serotype replacement: lessons from Haemophilus influenzae and prospects for Streptococcus pneumoniae. Emerging Infectious Diseases, 5- No. 3:336-345, 1999.

[6] M. Iannelli, M. Martcheva, and X.Z. Li. Strain replacement in an epidemic model with super-infection and perfect vaccination. Mathematical Biosciences, 195(1):23-46, May 2005.

[7] M. Martcheva. On the mechanism of strain replacement in epidemic models with vaccination. In Current Developments in mathematical biology. Proceedings of the Conference on Mathematical Biology and Dynamical Systems. (eds. K. Mahadavi, R. Culshaw \& J. Boucher), pp. 149-172. Hackensack, NJ: World Scientific Press., 2006.

[8] T. C. Porco and S. Blower. HIV vaccines: the effect of the mode of action on the coexistence of HIV subtypes. Mathematical Population Studies, 8(2):205229,2000 .

[9] P.G. Smith, L.C. Rodrigues, and P.E. Fine. Assessment of the protective effi- 
cacy of vaccines against common disease using case-control and cohort studies. International Journal of Epidemiology, 13:87-93, 1984.

[10] M.E. Halloran, M. Haber, and jr. I.M. Longini Interpretation and estimation of vaccine efficacy under heterogeneity. American J. Epidem., 136:328-343, 1992.

[11] S.M. Blower and A.R. McLean. Prophylactic vaccine, risk behavior change, and the probability of eradicating HIV in San Francisco. Science, 265- No. 5177:1451-1454, 1994.

[12] R. M. Anderson and R. M. May. Infectious diseases of humans: dynamics and control. Oxford Univ. Press, Oxford, 1991.

[13] A. Pugliese. Population models for diseases with no recovery. J. Math. Biol., 28:65-82, 1990.

[14] J.D. Murray. Mathematical Biology I: An Introduction. Springer-Verlag, 2002.

[15] N.F. Britton. Essential Mathematical Biology. Springer, 2003.

[16] W. Press, S. Teukolsky, W. Vetterling, and B. Flannery Numerical Recipes in C. The art of scientific computing. Cambridge University Press, 1992.

[17] V. Andreasen and A. Pugliese. Pathogen coexistence induced by density dependent host mortality. J. theor. Biol., 177:159-165, 1995.

[18] E. Massad, F.A.B. Coutinho, M.N. Burattini, L.F. Lopez, and C.J. Struchiner. The impact of imperfect vaccines on the evolution of HIV virulence. Medical Hypotheses, 66(5):907-911, 2006.

[19] H R Thieme and C Castillo-Chavez. How may infection-age-dependent infectivity affect the dynamics of HIV/AIDS? SIAM Journal on Applied Mathematics, 53:1447 - 1479, 1993.

[20] J.P. Hughes, G.P. Garnett, L.Koutsky. The Theoretical Population-Level Impact of a Prophylactic Human Papilloma Virus Vaccine. EPIDEMIOLOGY, 13(6): 631-639, 2002. 
[21] S. A. Levin and D. Pimentel. Selection for intermediate rates of increase in parasite-host systems. Amer. Nat., 117:308-315, 1981.

[22] J. Mosquera and F.R. Adler. Evolution of virulence: a unified framework for coinfection and superinfection. J. Theor. Biol.., 195:293, 1998.

[23] Troyer, R.M., Collins, K.R., Abraha, A., Fraundorf, E., Moore, D.M., Krizan, R.W., Toossi, Z., Colebunders, R.L., Jensen, M.A., Mullins, J.I., Vanham, G., Arts, E.J. Changes in human immunodeficiency virus type 1 fitness and genetic diversity during disease progression. J. Virol. 79:9006-9018 2005.

[24] C. Ball, M. Gilchrist, and D. Coombs Modeling Within-Host Evolution of HIV: Mutation, Competition and Strain Replacement Bull. Math. Biol. 69: 2361-2385, 2007. 